\title{
Increased Precision in the Intravascular Arterial Input Function with Flow Compensation
}

\section{Preface}

This is the peer reviewed version of the following article: "Bourassa-Moreau B, Lebel R, Gilbert G, Mathieu D, Lepage M. Increased Precision in the Intravascular Arterial Input Function with Flow Compensation. Magn Reson Med. 2019;82:1782-1795.", which has been published in final

form at DOI: $10.1002 / \mathrm{mrm} .27877$. This article may be used for non-commercial purposes in accordance with Wiley Terms and Conditions for Use of Self-Archived Versions. 


\section{$1 \quad$ Title page}

$2 \quad$ Title

3 Increased Precision in the Intravascular Arterial Input Function with Flow Compensation

$4 \quad$ Authors

5 Benoît Bourassa-Moreau ${ }^{1}$, Réjean Lebel ${ }^{1}$, Guillaume Gilbert ${ }^{2}$, David Mathieu $^{3}$, Martin Lepage ${ }^{1}$

$6 \quad$ Corresponding author: Martin Lepage

$7 \quad$ Complete contact information

8 For correspondence or reprints contact: Martin Lepage, Université de Sherbrooke, Centre 9 d'imagerie moléculaire de Sherbrooke, 3001 12th Ave. North, Sherbrooke, Québec, Canada, J1H 10 5N4. E-mail: martin.lepage@usherbrooke.ca

11 Text body word count: 5304 words

12 Institution information

13 1. Centre d'imagerie moléculaire de Sherbrooke, Département de médecine nucléaire et 14 radiobiologie, Université de Sherbrooke, 3001 12e Avenue Nord, Sherbrooke, Québec, Canada.

15 2. MR Clinical Science, Philips Healthcare Canada, Markham, Ontario, Canada.

16 3. Service de neurochirurgie, Département de chirurgie, Université de Sherbrooke, $300112 e$ 17 Avenue Nord, Sherbrooke, Québec, Canada. 


\section{Abstract}

Purpose: In this study, we investigate the effects of pulsatile flow and inflow on dynamic susceptibility-contrast MRI (DSC-MRI) intravascular arterial input function (AIF) measurement in human brain arteries and measure how they are affected by first-order flow compensation (FC).

Methods: A dual-echo single-shot EPI sequence with alternating FC gradients was used to acquire DSC-MRI data with electrocardiogram monitoring. The dynamic signal variations measured inside the middle- (MCA) and internal cerebral arteries (ICA) was associated to the pulsatile arterial blood velocities measured with a single-slice quantitative flow sequence 9 throughout the cardiac cycle.

Results: Major inverse correlations between intravascular signal and blood velocity were found for the standard SS-EPI sequence. FC reduces these correlated variations that contribute to signal physiological noise. It causes a significant two-fold increase of intravascular SNR in the MCA and the ICA, $(2.3 \pm 0.9, P=0.03)$ and $(2.0 \pm 0.9, P=0.04)$ respectively; and reduced phase standard deviation for the ICA $(0.72 \pm 0.14, P=0.004)$. The correction proposed in this work translates into a quantitative AIF with reduced noise in the ICA.

Conclusion: The physiological noise added by pulsatile flow and inflow for intravascular AIF measurement in the brain arteries is significantly reduced by FC.

Key Words: Dynamic susceptibility contrast MRI; flow compensation; blood flow; arterial input function; brain perfusion 


\section{Manuscript Body}

Introduction

Dynamic susceptibility contrast (DSC) can measure the changes in perfusion for brain tumors and cerebral ischemia. ${ }^{1}$ This dynamic imaging method measures the signal during contrast agent (CA) injection. The temporal signal change of each voxel is caused by CA effect on transverse relaxation $\left(\mathrm{T}_{2}^{*}\right)$ of the tissue and is weighted by tissue perfusion. DSC data analysis provides either a relative or an absolute quantitative perfusion measurement of cerebral blood flow (CBF) and volume (CBV). Absolute quantitative perfusion in clinical practice is currently limited because of the methodological complications and the variability of results reported in the literature. ${ }^{2} \mathrm{~A}$ cause for these variations is the error in the measured arterial input function (AIF), i.e. the tissue feeding artery concentration-time curve used for perfusion quantification. ${ }^{3}$

Accurate and precise AIF measurement is challenging. ${ }^{4} \mathrm{~A}$ first method is to extract the AIF from the signal inside a major artery. However, the $\mathrm{T}_{2}^{*}$-weighting for a typical single-echo DSC sequence is optimized to detect low CA concentrations in tissues. The resulting $\mathrm{T}_{2}^{*}$ decrease in major arteries is so important that the signal drops to the noise floor level at peak CA concentrations. This prevents accurate quantification of CA concentrations from intravascular blood signal measurement. An alternative method is to measure the AIF from tissues near the middle cerebral artery (MCA). ${ }^{5}$ The signal in these tissues remains well above noise and its changes are related to the AIF by the distant susceptibility effect of intravascular CA. Alternatively, the signal drop of a first echo with short TE in dual-echo EPI sequences is significant while remaining above noise therefore enabling the measurement of an AIF. Simulations have shown that both extra- and intravascular measurements are optimal for AIF selection with dual-echo EPI sequences. ${ }^{5}$

The growing interest in multi-echo DSC sequences for correction of CA extravasation provides an opportunity to choose between extra- and intravascular AIF measurements. An intravascular measurement has two advantages. First, it is not contaminated by background microcirculation that is known to broaden the measured extravascular AIF. ${ }^{6}$ Second, it allows absolute perfusion quantification with an AIF from the blood signal of the internal carotid artery (ICA), through a partial volume correction method (PVCM). ${ }^{7}$ This PVCM extracts the blood signal from the measured complex signal, which is contaminated by surrounding tissue. Such correction is possible for the ICA because its orientation and geometry are a good approximation of an infinite cylinder parallel to the main magnetic field. This condition nulls the tissue signal variations dependence with CA 
inside the artery. An absolute AIF measurement is not feasible in the MCA where the surrounding tissue signal depends on the CA-induced susceptibility effect outside the vessel with a strong dependence on the vessel distance, orientation, and geometry. Instead, intravascular signal measurements in the MCA provide a scaled version of the true AIF. ${ }^{4}$ Scaled AIF measurements provide relative perfusion metrics ( $\mathrm{rCBV}, \mathrm{rCBF}$ ) that are usually normalized by the white matter values contralateral to a lesion.

Both these scaled (MCA) and absolute (ICA) intravascular AIF measurements aim at measuring the signal of flowing blood. However, these measurements are contaminated by signal temporal variations inside major arteries that are partly caused by inflow and flow effects. ${ }^{8}$ The flow effects are caused by the motion of excited spins during the gradients applied between slice excitation and signal measurement. These blood spins experience an additional phase dispersion and offset that affect the complex signal. ${ }^{9}$ Inflow is caused by unexcited spins entering the slice. Its effects for AIF measurement have been described on the steady-state magnetization of 3D DCE sequences, ${ }^{10}$ and for the typical 2D multi-slices sequences used in DSC where the inflow of fresh spins partially replenishes the slice between each excitation. ${ }^{11}$ In this work, we extend these considerations by accounting for the pulsatile inflow between slice excitation and signal measurement.

The blood velocity in arteries changes during the cardiac cycle, which translates into repeated variations of the inflow and flow effects. For brain arteries and the typical single-shot EPI (SSEPI) sequence used in DSC, the pulsatile blood velocity remains approximately constant for the short duration of the echo train; every point in k-space of the slice is approximately acquired at this fixed velocity. On the other hand, DSC dynamic frames are sampled during various phases of the cardiac cycle, and each can be associated with a specific blood velocity. This effect was shown for a baboon artery where a correlation was found between the signal variations and the cardiac cycle phases for a single-echo SS-EPI DSC sequence. ${ }^{12} \mathrm{~A}$ first-order flow compensation (FC) in the slice selection direction limited these effects for AIF measurement. ${ }^{12,13}$ Such a method is not standard practice and, to our knowledge, an SS-EPI FC scheme generalized to all directions was not tested for the measurement of AIFs and is not available on clinical scanners.

The effects of pulsatile flow and excitation-to-measurement inflow on intravascular AlF measurement in human brain arteries are unknown. This study quantifies the correlation between the blood signal dynamic variations and its pulsatile velocity in the human MCA and ICA for a dual-echo SS-EPI sequence. These effects are characterized in several regions of the MCA to identify an ideal location for blood signal measurement. We show that FC extended to all encoding 
directions yields a two-fold increase of blood SNR in major arteries, and translates into the quantitative AIF measurement in the ICA.

\section{$3 \quad$ Methods}

\section{Acquisition}

Imaging was performed on an Ingenia 3.0T scanner with a 32-channel head receiver coil (Philips Healthcare, Best, Netherlands). Time of flight (TOF) angiography served to position the slices precisely over the MCA and to measure MCA and ICA segments angulation with respect to the imaging plane and $B_{0}$. $A$ high resolution $T_{1}$-weighted image was acquired for image registration. A single-slice quantitative flow sequence measured the subject specific pulsatile flow velocities perpendicular to the arteries for 15 trigger delays in the cardiac cycle. The maximal quantitative flow velocity in the artery region of interest (ROI) was calculated for each trigger delay. A custom single-slice 2D single-shot EPI dual gradient echo sequence was designed for intravascular signal measurements (Fig. 1a). Dynamic signal variations were assessed while recording the electrocardiogram (ECG) with automatic R-peak detection and acquisition trigger tagging; the frames were then sorted by ECG trigger delay. Images were acquired with (FC) or without (non-FC) first-order FC. For FC, gradient waveforms were added to provide gradient first moment nulling in all encoding directions (frequency encoding $\left(G_{f}\right)$, phase encoding $\left(G_{p}\right)$, and slice selection $\left(G_{s}\right)$ gradients). To compare the effect of $F C$ during the same dynamic acquisition, the gradient waveforms were alternated between the FC and the non-FC sequences. FC (odd frames) and non-FC (even frames) sequences were acquired alternately for 400 frames without CA injection. Other sequence parameters were as follows: Flip angle $=90^{\circ} ; \mathrm{FOV}=190 \times 226 \times 5$ $\mathrm{mm}^{3}$; acquired/reconstructed spatial resolutions $=3 \times 3 \times 5 \mathrm{~mm}^{3} / 2.8 \times 2.8 \times 5 \mathrm{~mm}^{3}$; reconstructed image matrix $=80 \times 80 ;$ SENSE factor $=2.3$; time resolution $=1.5 \mathrm{~s} ;$ SPIR fat saturation. Our sequence acquired spatial resolution is consistent with most DSC-MRI experiment, but the reconstructed spatial resolution is lower. This is caused by a smaller image matrix, and results in a reduced post-processing time without any loss of information.

\section{Cohort}

The study was approved by the institutional ethics committee and written informed consent was obtained from each subject. Fourteen young volunteers with an average age of $26 \pm 6$ years participated in this study ( 5 women and 9 men). The effect of FC on the final AIF and perfusion maps was measured in five subjects. To do so, the alternated FC/non-FC sequence was extended to multi-slice for the ICA measurements and brain coverage during Gd-DO3A-butrol injection $(0.05 \mathrm{mmol} / \mathrm{kg})$ with 180 frames post-injection and a flip angle of $30^{\circ}$. 


\section{Processing}

Data analysis was performed with Matlab R2016a (The MathWorks, Natick, MA). A Hessian based Frangi Vesselness filter ${ }^{14}$ and a threshold were applied to the TOF sequence to extract the subject arterial blood mask (Fig. 1b). The DSC slice position for MCA and ICA were overlaid over the arterial blood mask. The MCA and ICA segments angulation relative to the imaging plane was computed from 3D lines fitted over each straight portion of the left and right arteries.

Each DSC dynamic frame and slice was associated with its trigger delay, defined as the difference between its acquisition timestamp and the previous R-peak timestamp corrected for R$\mathrm{R}$ cardiac cycle period variations. The frames naturally cover the whole range of trigger delays due to the mismatch between the constant TR and the cardiac cycle period. Signal phase near the ICA was inspected for phase shifts and unwrapped when necessary. ${ }^{15}$ Arterial ROls were drawn manually to select the voxels with highest diastole signal magnitude within the arterial structure, as hyperintense blood signal is expected for this sequence with slight tissue saturation. For the MCA, the voxel selection was limited to a single voxel curve following the artery structure. This curve was split in four ROls based on the distance along the M1-segment (Fig. 1c). For the ICA, the four voxel of highest signal magnitude were selected for each artery (Fig. 1d). Signal magnitude is reported in arbitrary units (a.u.).

\section{Flow effects metrics}

Dynamic noise for a ROI signal magnitude and phase is defined as the standard deviation (SD) of its signal-time course obtained from repeated acquisitions. ${ }^{16}$ This noise is influenced by systematic dynamic signal variations such as the inflow and flow effects in vessels, patient motion, and other physiological variations. Signal to noise ratio (SNR) was computed for signal magnitude as the ratio of the mean value and the SD. Spearman correlation coefficient was calculated between blood flow velocities and signal-time courses (CORR). To do so, the arterial blood flow velocity acquired for 15 trigger delays was linearly interpolated at each trigger delay of the 200 frames from the FC or non-FC SS-EPI. CORR was calculated between these interpolated velocities and the signal-time courses. The ratio of FC to non-FC SNR $\left(R_{\mathrm{SNR}}=\mathrm{SNR}_{F C} / \mathrm{SNR}_{N C}\right)$ and phase $\mathrm{SD}\left(R_{\mathrm{SD}}=\mathrm{SD}_{F C} / \mathrm{SD}_{N C}\right)$ were taken as a measure of changes caused by $\mathrm{FC}$. Paired (CORR) and unpaired $\left(R_{\mathrm{SNR}}, R_{\mathrm{SD}}\right)$ Student's t-tests were used to assess whether the changes caused by $\mathrm{FC}$ are statistically significant $(P<0.05)$. 


\section{Perfusion experiment}

AIF concentration curves were computed from the phase of the first echo arterial ROI signal curve after applying a complex differences PVCM. ${ }^{7}$ Briefly, AIF quantification requires removing the surrounding tissue component from the measured signal. With increasing contrast agent concentration, blood signal follows a spiral-like trajectory in the complex plane caused by concurrent magnitude decrease $\left(\mathrm{T}_{2}^{*}\right)$ and phase change (susceptibility effects); whereas tissue signal is approximately constant as it is independent of the intravascular contrast agent for the ICA parallel with the main magnetic field. The PVCM fits a CA calibration curve spiral over the complex signal of the first pass time frames to find the constant tissue complex signal. The contribution of surrounding tissue is then removed from the measured signal. The corrected signal phase changes $(\Delta \theta(t))$ are related to the arterial concentration $\left(C_{A}(t)\right)$ by the equation:

$$
\Delta \theta(t)=\frac{1}{3} \gamma \mathrm{B}_{0} \chi_{m} C_{A}(t)
$$

with an assumed molar susceptibility of $\chi_{m}=3.209 \times 10^{-7} \mathrm{~mol}^{-1} .^{7}$ The calibration curve parameters are defined by this molar susceptibility and the quadratic $\Delta \mathrm{R}_{2, \text { blood }}^{*}$ relaxation rate for blood reported at $3 \mathrm{~T}::^{17}$

$$
\Delta \mathrm{R}_{2, \text { blood }}^{*}(t)=r C_{A}(t)+q\left(C_{A}(t)\right)^{2}
$$

with $r=0.4929\left(\mathrm{mM} \mathrm{s}^{-1}\right.$ and $q=2.6159\left(\mathrm{mM}^{2} \mathrm{~s}\right)^{-1}$.

Cerebral tissue concentration $\left(C_{T}(t)\right)$ was computed using the ratio of the first echo signal $\left(S_{1}(t)\right)$ over the second echo signal $\left(S_{2}(t)\right)$ :

$$
C_{T}(t)=\frac{1}{\mathrm{TE}_{2}-\mathrm{TE}_{1}} \ln \left[\frac{S_{1}(t) S_{2}\left(t_{0}\right)}{S_{2}(t) S_{1}\left(t_{0}\right)}\right] / r_{2}^{*}
$$

where $S_{1,2}\left(t_{0}\right)$ are the mean pre-injection signals of the first and second echoes, assuming a linear CA relaxivity of $r_{2}^{*}=87(\mathrm{~s} \cdot \mathrm{mM})^{-1} .{ }^{17}$ The tracer arrival time was measured for each voxel by computing the intersection between the pre-enhancement concentration and the exponential function that fits the first pass enhancement slope. CBF was computed by solving the basic tracer kinetic equation $\left(C_{T}(t)=C_{A}(t) \otimes(\mathrm{CBF} R(t))\right)$ with deconvolution using Tikhonov's regularization and cubic B-spline constraint on a tissue residue function $(R(t)) \cdot{ }^{18} \mathrm{CBV}$ was determined from the ratio of the areas under the tissue and arterial concentration time curves.

Registration of perfusion maps on the high resolution $\mathrm{T}_{1}$-weighted image was performed with Statistical Parametric Mapping (SPM12, revision number 6685). The gray (GM) and white matter 
(WM) masks were derived from the SPM12 tissue probability maps segmented over a subject's high resolution $\mathrm{T}_{1}$-weighted images and linearly interpolated over the perfusion maps. The mask was limited to the three top brain slices to avoid the signal loss near the prefrontal sinus. Perfusion values for FC against non-FC were plotted for all voxels of this mask and fitted with a linear regression $\left(y_{F C}=m \cdot x_{n o n-F C}+b\right)$. The percent deviation of its slope from the line of equality $\left(D_{F C}=100 \%(m-1)\right)$ is taken as a measure of global agreement between FC and non-FC. The SD of $D_{F C}$ was computed from the $95 \%$ confidence interval of the fitted slope.

\section{Results}

\section{Dynamic variations of blood signal}

Slice positioning for intravascular dynamic measurements and evaluation of the vessel shape were based on TOF data (Fig. 1b). The associated manual ROI selection is shown in the SS-EPI image of one subject for MCA (Fig. 1c) and ICA (Fig. 1d).

We evaluated whether the blood signal variations in these ROls were correlated with blood velocity. The signal-time curves before CA injection are shown in Fig. 2a. Dynamic gradients waveforms alternation allows to extract both non-FC and FC curves from the same dynamic acquisition. Increased SNR can already be appreciated from the FC curve. ECG trigger delay was associated with each dynamic frame during post-processing (Fig. 2c). Sorting signal-time curves by trigger delay unveils signal variations that have a large inverse correlation with pulsatile blood velocity (CORR $=-0.86, P<0.001)$ (Fig. 2b). FC substantially decreases the correlation between signal and blood velocity $(\mathrm{CORR}=-0.38, P<0.001)$; this translates into a reduction of the apparent noise in the corresponding curve in Fig. 2a. Non-FC correlation measurements were highly significant $(P<0.001)$ for all subjects. The only non-significant correlations $(P>0.05)$ were found for FC measurements in the ICA for three subjects. This is expected for these cases since the correlation was approaching zero $(|\mathrm{CORR}|<0.11)$. The $p$-value indicates the significance level of the correlation and could evaluate the effect of FC, but we report only the more intuitive correlation value for the remaining work.

Examples of the sorted signal curves and their associated flow effect metrics for a representative individual subject are shown in Figs. 3 and 4 for the MCA and the ICA, respectively. In Fig. 3, intravascular signal variations are highly correlated with blood velocity for all ROls. FC partially eliminates this correlation for ROI 3, which results in a 2.3x increase of SNR. The signal variations for ROls 1, 2 and 4 cannot be removed by FC. For this reason, ROI 3 in the MCA was chosen for further evaluation of the effects of FC. In Fig. 4, ICA magnitude and phase signal 
variations are inversely correlated with blood velocity. FC alleviates most of the correlation between signal magnitude and velocity, but the effect is limited for the signal phase in this subject. The statistical differences between FC and non-FC are not reported for these individual subject data but are reported in the following results for the whole cohort.

The $R_{\mathrm{SNR}}, R_{\mathrm{SD}}$, and correlation metrics for MCA ROI 3 and ICA were calculated for every subject (Fig. 5). FC results in a significant two-fold increase of blood SNR in the MCA and the ICA, $(2.3 \pm 0.9, P=0.03)$ and $(2.0 \pm 0.9, P=0.04)$ respectively (Fig. 5a); and reduced phase SD for the ICA $(0.72 \pm 0.14, P=0.004)$ (Fig. $5 b)$. Signal to velocity correlation changes were only significant for ICA signal magnitude $(P=0.01)$ and phase $(P=0.002)$ (Fig. 5c).

\section{Perfusion experiment}

We next assessed whether the FC pre-injection noise reduction translates into improved AIF and perfusion measurements. Images were acquired with the alternated sequence during and after CA injection. The PVCM corrected phase-based AIF of all subjects are reported in Fig. 6. It is immediately apparent that data points acquired at peak velocity with the non-FC scheme (open circles) are an important source of noise. Reduced noise in the FC signal phase (open squares) translates to the AIF, seven out of ten cases showed reduced sharp temporal variations associated with noise. The only exceptions are the AIFs of panels $\mathrm{g}$, $\mathrm{i}$ and $\mathrm{j}$ where FC does not provide apparent noise reduction. The major AIF non-FC to FC differences of Fig. $6 \mathrm{~h}$ and $\mathrm{j}$ are caused by variations of the solution of the PVCM. The PVCM is detailed for two AIFs in Fig. 7 for non-FC and FC acquisitions. For both AIFs, the signal in the complex plane for non-FC data (Fig. $7 a, c)$ appears to be noisier than their FC counterparts (Fig. 7b,d) as judged from the reduced contrast between the first pass time frames (black dots) and the other time frames (gray dots). Major differences in the fitted calibration curve for the non-FC and FC AIF of subject 5 (Fig. 7c,d) result in a truncated AIF peak for the non-FC data and a shift of the concentration values at $85 \mathrm{~s}$ (Fig. 7f). This shift matches major subject motion in the raw dynamic images resulting in the exclusion of this dataset from perfusion maps measurements.

We examined the effect of FC on perfusion maps. The AIF from the left ICA (Fig. 6f) was chosen for the perfusion measurement of Fig. 8. Qualitatively comparable CBF and CBV maps are obtained from FC and non-FC data (Fig. 8ac). The voxel by voxel comparison of Fig. 8b and $8 \mathrm{~d}$ highlights the global differences between the two sequences. The agreement between FC and non-FC values are fair and show a slight deviation above and below the line of equality for CBF and $\mathrm{CBV}$, respectively. Table 1 shows the perfusion results for all subjects AIFs with average values of CBF and CBV over GM and WM ROls and the $D_{F C}$. FC increases the CBF for five cases 
and reduces the CBV for six cases as can be seen by the significant changes highlighted in the $D_{F C}$ column. The perfusion results from an AIF with major non-FC to FC PVCM differences is underlined.

\section{Discussion}

\section{Dynamic variations of blood signal}

Pulsatile constant-velocity flow effects are a major cause of blood signal variations for intravascular AIF measurement in the MCA. FC fully compensates these effects for the center of k-space with increasing residual flow effects along the SS-EPI echo train. Overall, pulsatile blood signal variations in DSC-MRI are partially recovered by FC. This was shown theoretically in the tube simulation of the supporting information (Fig. S1) and confirmed by the increased SNR in clinical pre-injection measurements (Fig. 5a).

Strong inverse correlations between MCA and ICA intravascular signal and blood velocity were found for standard (non-FC) SS-EPI sequence (Fig. 5c). FC reduces the negative correlation contribution of constant-velocity flow effect; the CORR values become closer to zero. The correlation of blood signal with blood velocity causes random fluctuations in the non-FC signaltime course. Compensating for flow effects yields a significant two-fold increase of SNR and a significant decrease of phase SD for the ICA (Fig. 5ab). As FC corrects only for constant-velocity flow effects, it shows that these effects are a major source of dynamic noise for intravascular AlF measurement. A weaker inverse correlation is found for the ICA signal phase than for the signal magnitude (Fig. 5c). Signal phase variations have been linked to the respiratory cycle by others, ${ }^{12}$ and could explain increased uncorrelated noise sources in phase data. Similar results for the FC effects on pre-injection intravascular signal are reported for the perfusion sequence with a $30^{\circ}$ flip angle (Supporting information - Fig. S2).

Other sources of velocity-correlated signal variations could not be recovered by FC, i.e. inflow and higher order flow effects. The methods in this work were developed to reduce these effects. First, careful slice placement is required to limit unexcited blood inflow for the in-slice MCA AIF. The MCA has an approximate diameter of $3 \mathrm{~mm}$ in this region, which allows margins for blood excitation with the $5 \mathrm{~mm}$ slice of the SS-EPI sequence while minimizing tissue signal in the voxel volume. During sequence planning, the $5 \mathrm{~mm}$ slice was positioned to cover both left and right MCA M1-segment on the TOF images. Arterial masks show good slice placements for most of the subjects. However, the blood mask reveals a small amount of unexcited blood in ROI 2 for one subject (Supporting information - Fig. S3). The excited slice is not thick enough to cover the 
entire blood from this highly curved MCA. Considering the actual slice profile, a larger amount of blood is expected to have an imperfect flip angle. The inflow of unexcited blood in ROI 3 could be the cause of the velocity-correlated signal variations that cannot be recovered by FC. Increasing slice thickness could solve this issue at the cost of higher partial volume effect.

Next, using a single-slice acquisition ensured that the magnetization of inflowing blood was in a fully relaxed state. Multi-slice acquisition could cause additional blood $\mathrm{T}_{1}$ effects by exciting upstream blood; such effect would also be correlated with blood velocity. ${ }^{11}$ This can well be the case for MCA measurements with full brain coverage. This effect can be avoided by considering the transit time of blood in the vessels upstream when choosing the number of slices prior to the MCA and by careful selection of the slice ordering scheme.

Finally, selection of voxels at the end of the straight part of the M1-segment can reduce flowrelated effects. Indeed, flow effects are mainly of constant-velocity when the infinite cylinder approximation is valid for the distance traveled by blood during TE. ROI 3 (Fig. 1c) fulfilled this condition for four out of five subjects with MCA measurements. Signal variations could not be decreased in regions where inflowing blood was not excited (ROI 1) or when inflow of excited blood traveling through vessel curvature are expected to cause higher order flow effects (ROI 2 and 4). In a typical DSC experiment, automatic or manual AIF voxel selection does not rely on TOF data or ECG trigger delay measurements. In this case, the ideal voxels to reduce flow-related effects with FC can be identified as voxels with increased dynamic SNR for the intravascular FC signal that are located three quarters along the M1-segment of the MCA. Fewer voxels are available for AIF measurements in the ICA perpendicular to the imaging slice. Unfortunately, careful voxel selection cannot account for inflow and higher order flow effects that may still occur. In such a case, careful placement of the slice upstream of a straight section of the ICA and perpendicular to the vessel should help reduce these effects.

Non-FC signal was positively correlated $($ CORR $>0)$ with velocity in the ICA for one subject (Fig. 5c). Accordingly, $R_{\mathrm{SNR}}$ was lower than 1 for this subject (Fig. 5a). Flow-induced dephasing always causes a negative correlation with velocity and therefore cannot have caused this positive correlation. Inflow effects in the ICA could be the cause of this observation. Following this idea, inter-individual differences of the arterial physiology may explain the variable level to which $R_{\mathrm{SNR}}$ can be increased by FC (Fig. 5). Indeed, we have shown for MCA that FC fails for a subject with increased vessel curvature (Supporting information - Fig. S3) where no voxel allows for blood inflow from a straight segment upstream. This could also be true for a curved ICA downstream of 
the excited slice. Also, it is known that flow effects in SS-EPI will have a strong variation with vessel orientation relative to $G_{p}{ }^{8}$

Altogether, our results provide additional insights on whether the AIF should be measured inside or outside the MCA. Simulations by others have shown that intravascular AIF measurement could be optimal for the short TE of multi-echo EPI sequences to avoid blood signal saturation during bolus passage. ${ }^{5}$ However, these simulations did not consider SNR degradation by pulsatile blood flow effects. Our results show that FC partially recovers these effects but that careful slice placement over the MCA is required. This might not be possible for routine DSC experiments without reference TOF data or when multi-slice coverage is already constrained by FOV requirements. Conversely, no pulsatile flow dependent noise was found for the tissues near the arteries. AIF measurement in the tissues surrounding the MCA provides high SNR dynamic signal for relative AIF measurement.

The signal magnitude and phase trigger delay variations found for the ICA is consistent with previously reported findings for the baboon aorta. ${ }^{12}$ This previous work reported results for a FC SS-EPI sequence where the FC was restricted to the slice selective gradient $\left(G_{s}\right)$. To minimize the trigger delay dependent signal variations, R-wave triggered acquisition during the low velocity diastole was proposed for intravascular AIF measurement. The TR would thus be continually adjusted to match the slightly variable cardiac cycle period, which changes the $\mathrm{T}_{1}$-weighting throughout the experiment. This would cause a variation of CA extravasation effects and is not practical for brain tumor imaging. Some of these uncorrected variations might be caused by uncompensated flow in the slice plane. In our study, the ICAs were misaligned with $G_{s}$ by $(11 \pm 7)^{\circ}$ providing a transverse component of flow that is FC by in-slice motion correction $\left(G_{f}\right.$ and $\left.G_{p}\right)$ additionally to $G_{s}$. For the ICA, residual flow-related noise is small compared to other noise sources after FC. The angulation and geometry of the abdominal aorta were not reported in the baboon measurements. ${ }^{12}$ The relative improvement of $F C$ in all directions compared to a $G_{s}-$ only FC is unknown for the ICA. A first level of approximation detailed in Section D of the Supporting Information suggests that FC in all directions is superior for poorly aligned ICA; FC recovers AIF concentration variations of up to $10 \%$ of the peak concentration caused by the transverse component of flow.

\section{Perfusion experiment}

Signal-time curves are affected by noise caused by the variable blood velocity thereby degrading the intravascular AIF measurement in perfusion experiments. Constant-velocity flow effects directly affect phase-based AIF measurements. Points with largest deviations are 
associated with frames measured at peak velocity (Fig. 6). FC efficiently reduces these concentration variations. Visible noise reduction can be appreciated for seven out of ten FC AIFs.

CBF and CBV changes caused by FC are reported, but it is not clear whether these changes increased the accuracy of the perfusion values as the true values are unknown. Nevertheless, our methodology reduces noise in intravascular signal which is expected to improve the shape and precision of the AIF, and to directly influence the quality of perfusion maps. ${ }^{3,13}$ Flow induced signal variations consisted of a drop of signal phase at peak blood velocity. This results in lower average AIF concentrations for non-FC data thus a lower area under the AIF curves. This explains the decrease of CBV with FC observed for most subjects (Table 1). The effect of FC on CBF is more complex. It will depend on how flow effects change the shape of the AIF first pass and how these changes translate through the regularized deconvolution required for CBF calculation. In addition, the drop of AIF concentration caused by peak flow effects occurs during random time frames. We note an increase of CBF for two out of three subjects (Table 1) for whom flow effects occur during the first pass (Fig. 6a,b,c,d,e,f).

Major differences in the fitted spiral calibration curve from the PVCM were found for two AIFs with either higher (Fig. 6j) or truncated (Fig. 6h) peak AIF for non-FC data. Higher non-FC AIF peak is the cause of major CBF and CBV increases reported in Table 1 (underlined data). The PVCM differences could be attributed to the additional noise in non-FC complex signal. The PVCM relies on the magnitude and phase of the signal; both of which are reduced by flow effects at peak velocity. This could result in PVCM failure for non-FC data. However, other limitations reduced the performance of PVCM in this study and could be responsible for differences in the fitted calibration curves. First, SS-EPI sequences are sensitive to the signal displacement induced by magnetic field changes during CA passage. ${ }^{19}$ With our SS-EPI sequence bandwidth of 53.2 $\mathrm{Hz} /$ pixel, we expect a displacement of 1 pixel at the peak arterial concentration of $4 \mathrm{mM}$. This shift might limit the performance of the PVCM algorithm in correcting the AIF. Next, PVCM relies on the approximation that the ICA is aligned with $\mathrm{B}_{0}$. For our experiment, the angle between the ICA and $B_{0}$ was $(10 \pm 6)^{\circ}$. This allows minor $C A$ susceptibility effects outside the vessel that can reduce the efficiency of PVCM. Last, concentration calibration curves for ICA AIF relied on $\mathrm{R}_{2}^{*}$ relaxivity ${ }^{17}$ and molar susceptibility ${ }^{7}$ taken from literature for Gd-DPTA. These values could differ for the $\mathrm{Gd}$ DO3A-butrol used in this study. These constants change the shape of the fitted calibration curve in the PVCM procedure, which could affect the measured tissue signal contribution, and scale the phase-based AIF. 
Absolute estimates of gray matter CBF for our subjects are within the range reported at 3T but our CBV values are lower. ${ }^{2}$ The ROls were derived automatically from GM and WM segmentation and were thus user-independent. A drawback is the inclusion of all voxels regardless of their perfusion variations, their proximity to major blood vessels, or other surrounding tissues; this results in a high SD for CBF and CBV in Table 1. GM CBV would be increased if one manually delineated ROIs to exclude voxels with apparent partial volume effects with surrounding WM.

\section{Other sequences}

This work focused on pulsatile flow and inflow effects specifically for 2D SS-EPI DSC sequences. Such effects are to be considered for intravascular AIF measurements with other dynamic sequences.

Multi-echo single-shot spiral-out sequences ${ }^{20}$ are expected to be robust to flow effects because their k-space trajectory preserves the properties that provide flow immunity reported for multi-shot spiral-out imaging. ${ }^{8}$ Multi-shot EPI (MS-EPI) sequences are known to be sensitive to flow effects with major distortions and ghosting. ${ }^{8}$ The fast k-space sampling of 2D MS-EPI sequences might lead to important velocity variations between k-space time frames, hence to pulsatile signal variations. However, if the MS-EPI k-space is fully sampled over a full cardiac cycle, this effect might appear as a constant blood signal offset where each time frame averages the cardiac cycle velocity variations. This condition should be met for 3D MS-EPI sequences.

For DCE-MRI sequences, the inflow effects have previously been studied. ${ }^{10}$ However, the incidence of flow effects and the pulsatile blood flow of the cardiac cycle were not considered. Flow effects are known to cause signal displacement in SPGR sequences such as in typical DCE sequences. ${ }^{9}$ For pulsatile flow in 3D DCE sequences, the k-space is filled during the whole dynamic scan time covering a wide range of velocities. Based on our simulations, the flow-induced signal displacement remains constant throughout the dynamic frames and is not a major source of signal variation (Supporting information - Fig. S4). This constant signal displacement partly overlaps with the blood signal and surrounding tissue and increases the partial volume effect. It can also lead to misalignment of the arterial signal with the initial blood $\mathrm{T}_{1}$ map. The signal displacement might differ between DCE data and $\mathrm{T}_{1}$ map if another sequence type is used or if gradient echo sequence parameters such as bandwidth and resolution are not identical. 


\section{Conclusion}

In summary, this study demonstrated the performance of a methodology based on FC to improve the SNR of intravascular AIFs. For intravascular AIF measurement in the MCA, the methodology includes FC, a careful slice placement and voxel selection at the end of the straight portion of the M1-segment. The reduced noise in FC signal magnitude and phase increases the precision of PVCM for absolute AIF measurement in the ICA. This could help further improve the reproducibility of quantitative perfusion measurements.

\section{$8 \quad$ Acknowledgements}

This work was supported by a grant from the Fonds de recherche du Québec (FRQ) - Nature et technologies (2018-PR-206157). ML is member of the FRQ-Santé-funded Centre de recherche 11 du CHUS. BBM acknowledges scholarship from National Sciences and Engineering Research 12 Council of Canada.

\section{Conflict of Interest}

14 M.L. is member of the FRQ-Santé-funded Centre de recherche du CHUS. 


\section{References}

1. Welker K, Boxerman J, Kalnin A, Kaufmann T, Shiroishi M, Wintermark M. ASFNR recommendations for clinical performance of MR dynamic susceptibility contrast perfusion imaging of the brain. Am J Neuroradiol. 2015;36:E41-E51.

2. Knutsson L, Ståhlberg F, Wirestam R. Absolute quantification of perfusion using dynamic susceptibility contrast MRI: Pitfalls and possibilities. Magn Reson Mater Phys. 2010;23:121.

3. Calamante F, Connelly A. DSC-MRI : How accurate does the arterial input function need to be in practice? Proc Int/ Soc Mag Reson Med. 2007;15:593.

4. Calamante F. Arterial input function in perfusion MRI: A comprehensive review. Prog Nucl Magn Reson Spectrosc. 2013;74:1-32.

5. Bleeker EJW, van Buchem MA, van Osch MJP. Optimal location for arterial input function measurements near the middle cerebral artery in first-pass perfusion MRI. J Cereb Blood Flow Metab. 2009;29:840-852.

6. Thornton RJ, Jones JY, Wang ZJ. Correcting the effects of background microcirculation in the measurement of arterial input functions using dynamic susceptibility contrast MRI of the brain. Magn Reson Imaging. 2006;24:619-623.

7. van Osch MJP, Vonken EPA, Viergever MA, van der Grond J, Bakker CJG. Measuring the arterial input function with gradient echo sequences. Magn Reson Med. 2003;49:10671076.

8. Nishimura DG, Irarrazabal P, Meyer CH. A Velocity k-Space Analysis of Flow Effects in Echo-Planar and Spiral Imaging. Magn Reson Med. 1995;33:549-556.

9. Nishimura DG, Jackson JI, Pauly JM. On the nature and reduction of the displacement artifact in flow images. Magn Reson Med. 1991;22:481-492.

10. Roberts C, Little R, Watson Y, Zhao S, Buckley DL, Parker GJM. The effect of blood inflow and $\mathrm{B}(1)$-field inhomogeneity on measurement of the arterial input function in axial 3D spoiled gradient echo dynamic contrast-enhanced MRI. Magn Reson Med. 2011;65:108119.

11. Calamante F, Vonken EJPA, van Osch MJP. Contrast agent concentration measurements affecting quantification of bolus-tracking perfusion MRI. Magn Reson Med. 2007;58:544- 
553.

12. Akbudak E, Conturo TE. Arterial input functions from MR phase imaging. Magn Reson Med. 1996;36:809-815.

13. Kotys MS, Akbudak E, Markham J, Conturo TE. Precision, signal-to-noise ratio, and dose optimization of magnitude and phase arterial input functions in dynamic susceptibility contrast MRI. J Magn Reson Imaging. 2007;25:598-611.

14. Kroon D-J. Hessian based Frangi Vesselness filter.https://www.mathworks.com/matlabcentral/fileexchange/24409-hessian-basedfrangi-vesselness-filter. MATLAB Central File Exchange.

15. Maier F, Fuentes D, Weinberg JS, Hazle JD, Jason Stafford R. Robust phase unwrapping for MR temperature imaging using a magnitude-sorted list, multi-clustering algorithm. Magn Reson Med. 2015;73:1662-1668.

16. Dietrich O, Raya JG, Reeder SB, Reiser MF, Schoenberg SO. Measurement of signal-tonoise ratios in MR images: Influence of multichannel coils, parallel imaging, and reconstruction filters. J Magn Reson Imaging. 2007;26:375-385.

17. Kjølby BF, Østergaard L, Kiselev VG. Theoretical model of intravascular paramagnetic tracers effect on tissue relaxation. Magn Reson Med. 2006;56:187-197.

18. Larsson HBW, Hansen AE, Berg HK, Rostrup E, Haraldseth O. Dynamic contrastenhanced quantitative perfusion measurement of the brain using T1-weighted MRI at 3T. J Magn Reson Imaging. 2008;27:754-762.

19. Rausch M, Scheffler K, Rudin M, Radü EW. Analysis of input functions from different arterial branches with gamma variate functions and cluster analysis for quantitative blood volume measurements. Magn Reson Imaging. 2000;18:1235-1243.

20. Paulson ES, Prah DE, Schmainda KM. Spiral Perfusion Imaging With Consecutive Echoes (SPICE ${ }^{\mathrm{TM}}$ ) for the Simultaneous Mapping of DSC- and DCE-MRI Parameters in Brain Tumor Patients: Theory and Initial Feasibility. Tomography. 2016;2(4):295-307. 
a.

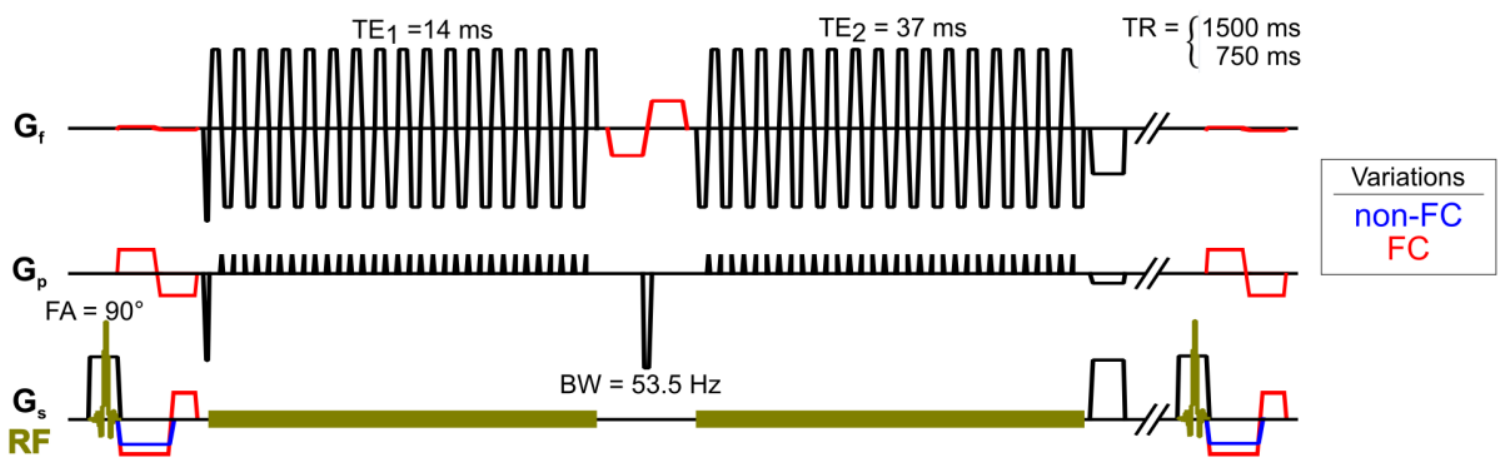

b.
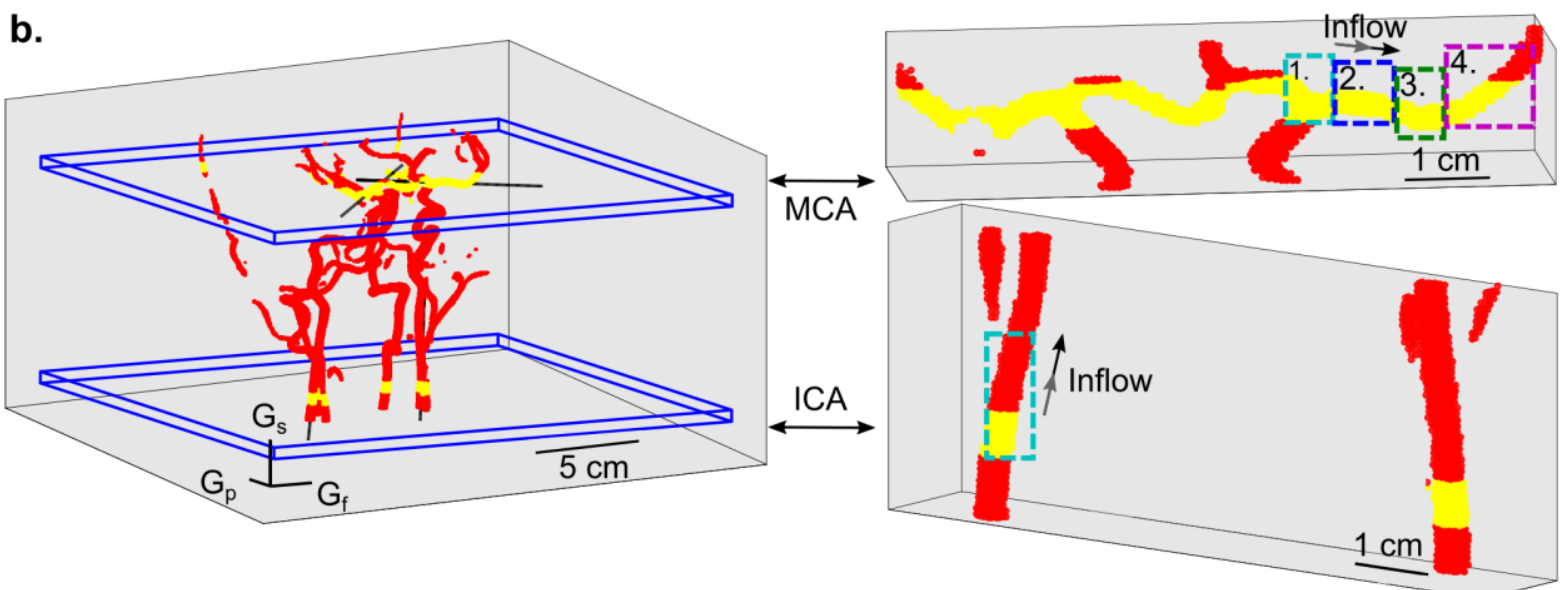

c.

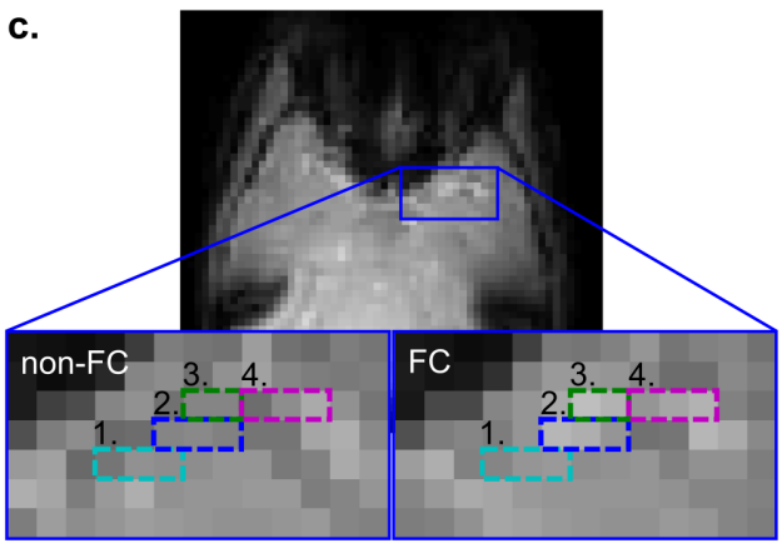

d.

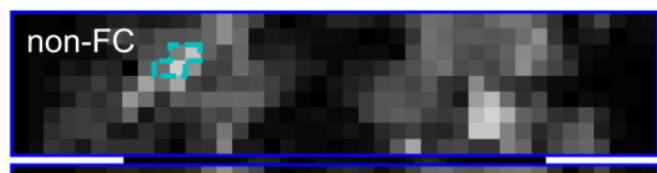

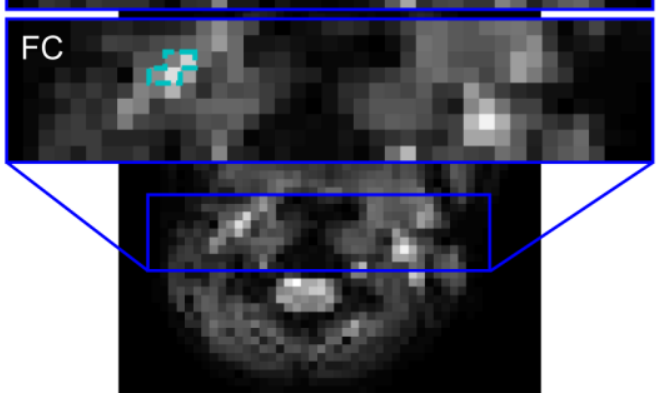

FIG. 1. DSC sequence diagram with slice and ROI placement for measuring arterial blood signal. (a) Dual-echo single-shot EPI FC and non-FC sequence diagram. (b) Arterial blood mask derived from TOF sequence (yellow/red) with DSC slice position for MCA and ICA (blue boxes). Blood mask within the excited slice is shown in yellow. Initial TOF images allow for the positioning of the $5 \mathrm{~mm}$ slice during the acquisition protocol. The blood mask in the left and right M1-segment of the MCA is completely included within the excited slice. The black lines are the linear regression 
of the straight part of each vessel used to measure the vessel orientation with encoding gradients $2\left(G_{f}, G_{p}, G_{s}\right)$ and $B_{0}$. The enlarged sections of the MCA and the ICA are annotated with gray and 3 black arrows to show the expected length of inflow in systole and diastole, respectively. For 4 example, the inflow effect extends for $5 \mathrm{~mm}$ (diastole) to $8 \mathrm{~mm}$ (systole) with the MCA based on 5 the first echo TE (14 ms) and blood velocities (33 to $59 \mathrm{~cm} / \mathrm{s}$ from subject data). ROls of the 6 arteries are defined in the DSC images (dashed boxes in (c) MCA and (d) ICA first echo DSC 7 images). Reporting these ROls on the TOF arterial blood mask (b), one can appreciate the extent 8 of blood inflow and vessel curvature expected for each ROI. 
a.

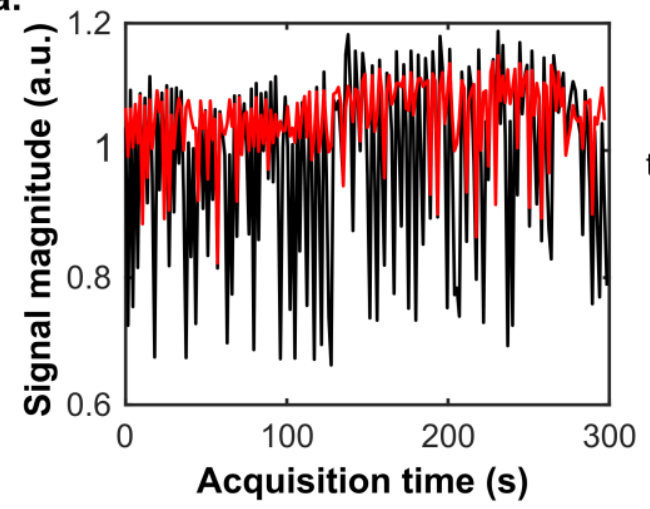

b.

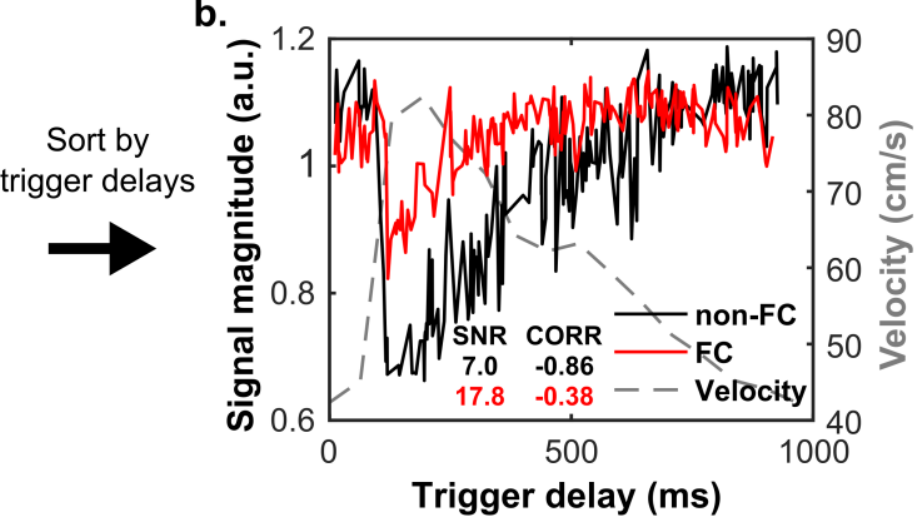

c.

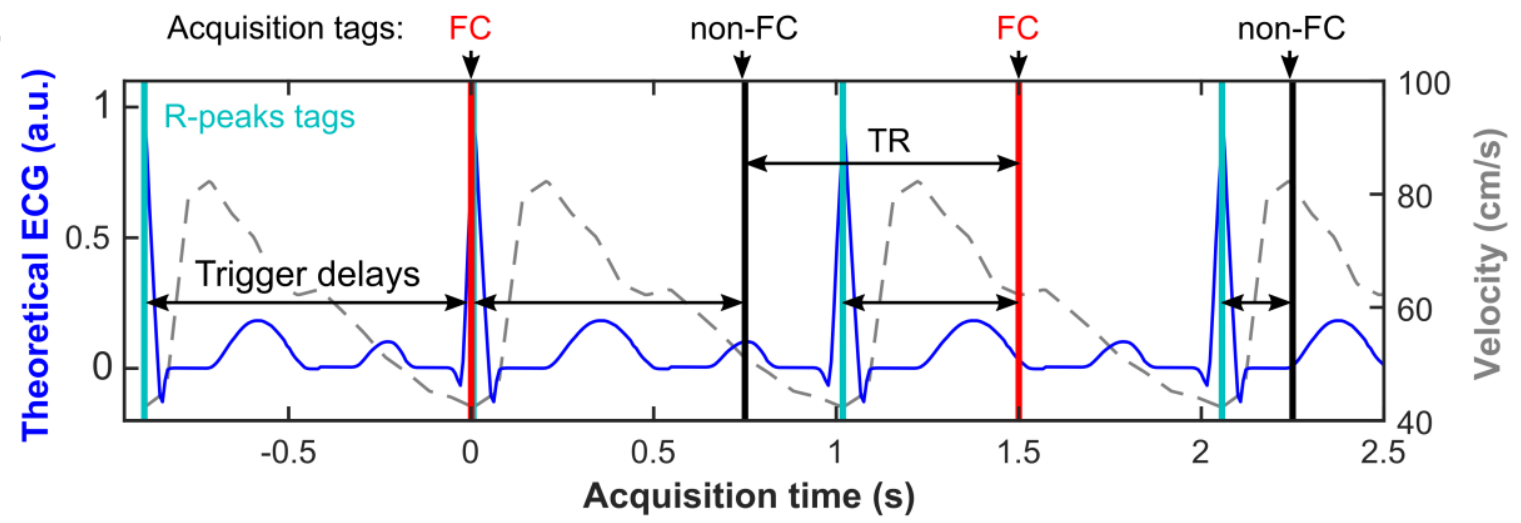

FIG. 2. Sorting of the dynamic frames by trigger delay. (a) Unsorted signal magnitude-time curve from a voxel of MCA ROI 3 (Fig. 1c) where the even frames are non-FC and odd frames are FC. (b) Signal magnitude from (a) sorted by associated trigger delays. SNR and correlation between signal and velocity (CORR) measured for each curve are shown on this panel. (c) An example of the trigger delay measurement for the first four dynamic frames from ECG R-peak and acquisition timestamps (FC and non-FC) that are logged by the MR scanner. The measured MCA blood flow velocity (dashed gray line) was added to enable direct comparison. 

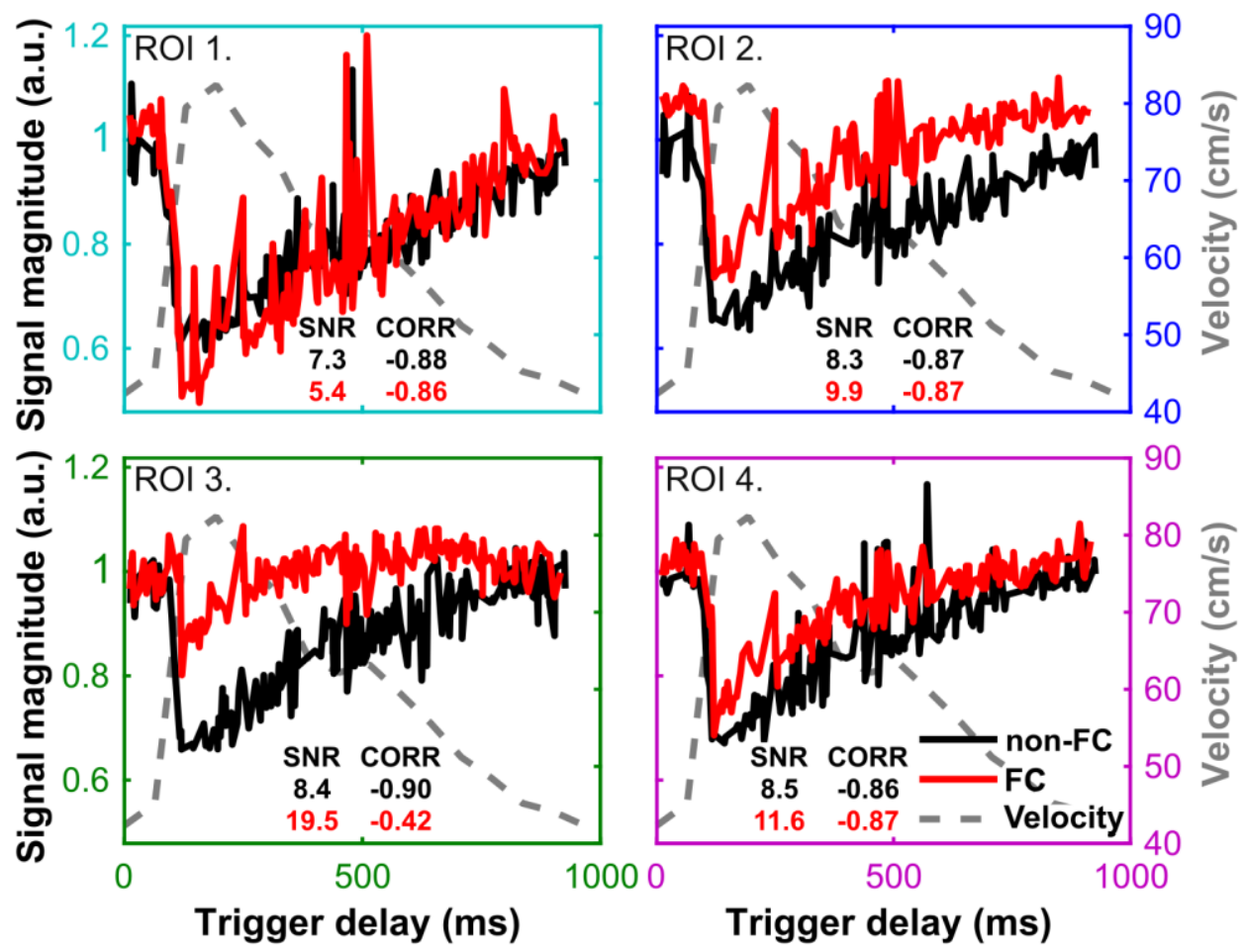

FIG. 3. Dynamic first echo signal sorted by trigger delay for each ROI in the MCA (Fig. 1c) for FC and non-FC SS-EPI sequences with measured blood flow velocity (dashed curve). SNR and correlation between signal and velocity (CORR) are indicated for each ROI. Non-FC signal variation is highly correlated with blood flow velocity in all sections of the MCA. FC increases the SNR and reduces the absolute correlation mostly for ROI 3. This region corresponds to the last section of the straight part of the MCA (see Fig. 1b). FC is effective in this region because flow effects are mainly due to constant-velocity, i.e. the blood inflow between excitation and measurement comes from upstream excited blood flowing in a straight line. 


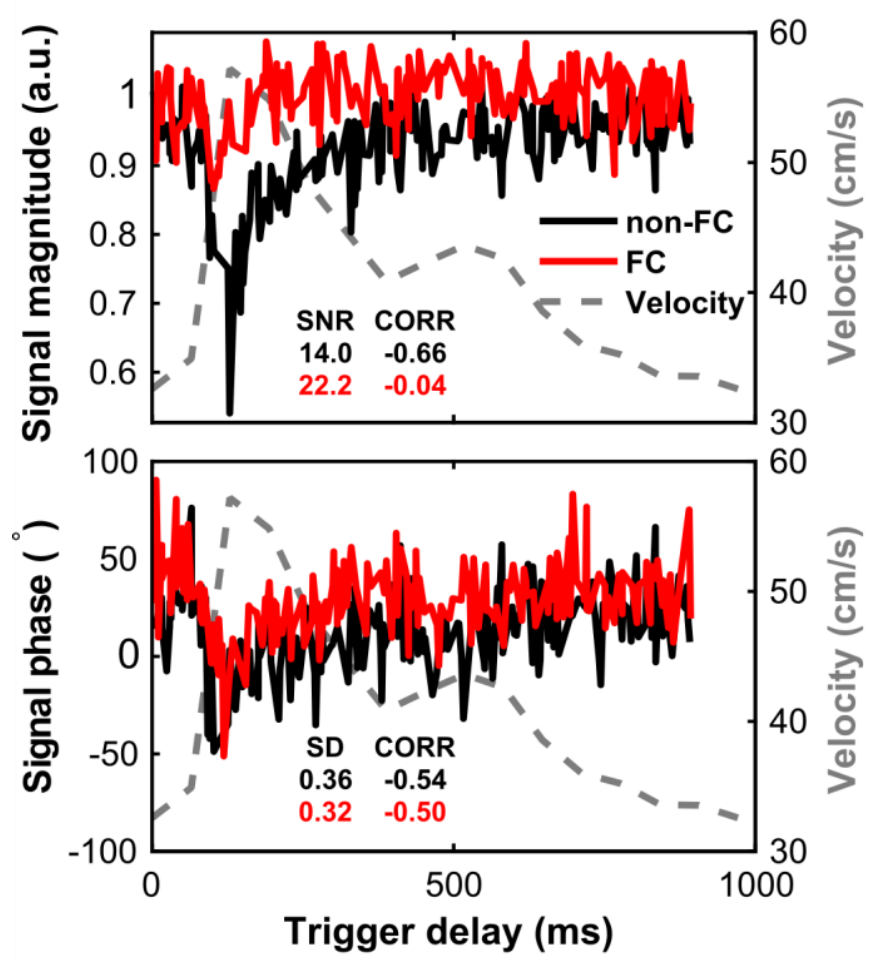

2 FIG. 4. Dynamic signal magnitude and phase of the first echo sorted by trigger delay for the ICA 3 ROI (see Fig. 1d) for FC and non-FC SS-EPI sequences with measured ICA blood flow velocity 4 (dashed curve). SNR, phase SD and correlation between signal and velocity (CORR) are 5 indicated for each curve. Non-FC magnitude and phase signal variations are inversely correlated 6 with the blood flow velocity in the ICA. FC increases the SNR and reduces the correlation between 7 signal magnitude and velocity. The component of the dynamic noise associated with constant8 velocity flow effects is reduced by FC. 

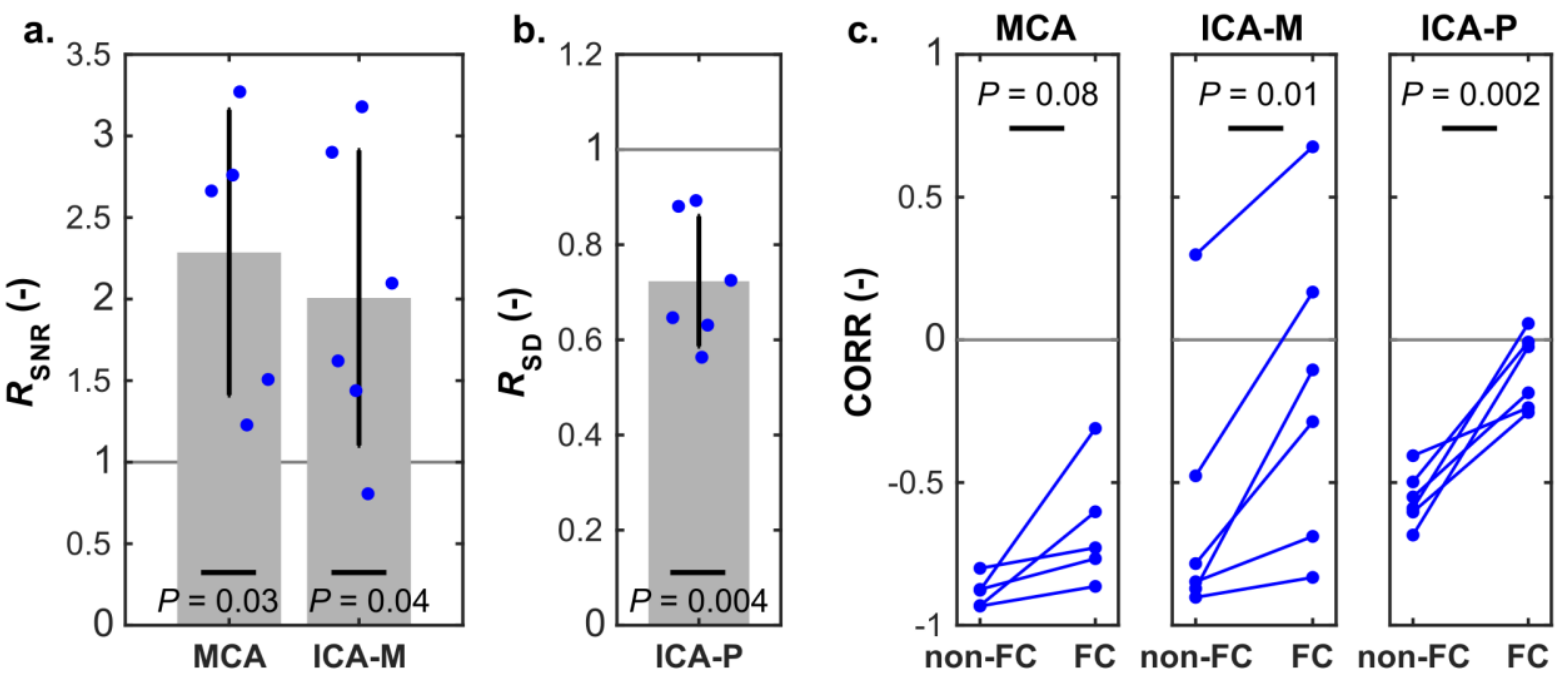

FIG. 5. Effect of FC on SS-EPI (a) SNR ratio ( $\left.R_{\mathrm{SNR}}\right)$, (b) phase SD ratio $\left(R_{\mathrm{SD}}\right)$, and (c) correlation 4 between signal and flow velocity (CORR). These metrics are compiled for all subjects MCA (N=5) 5 and ICA (N=6). ICA-M and ICA-P indicate the metrics calculated for ICA signal magnitude and 6 phase, respectively. $p$-values are shown for all datasets. 


\section{RIGHT}

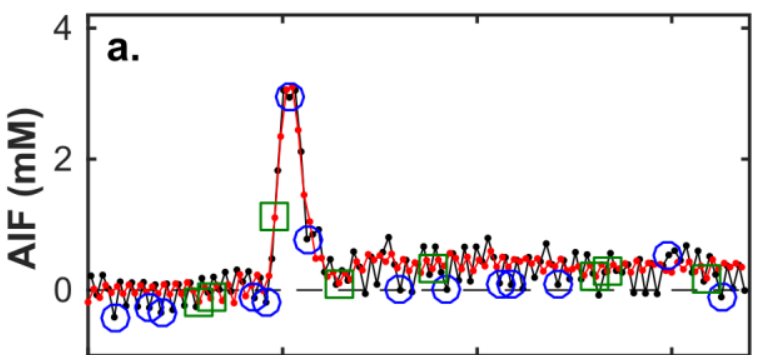

ㄴ.

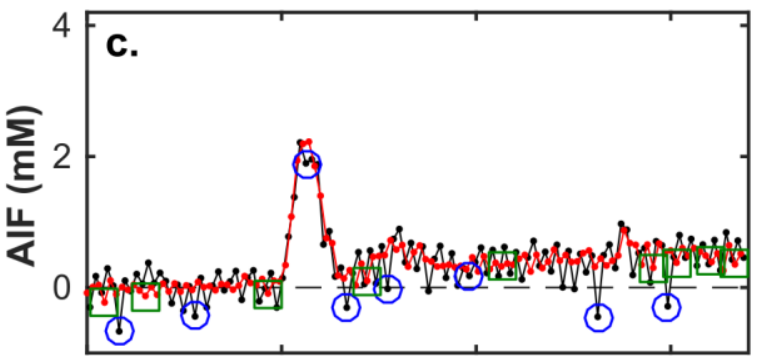

क)

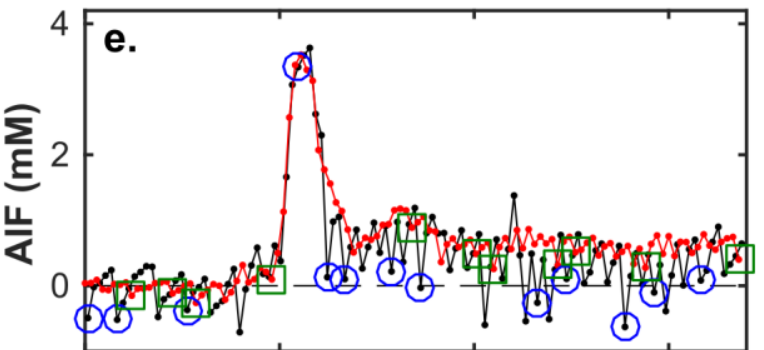

ำ

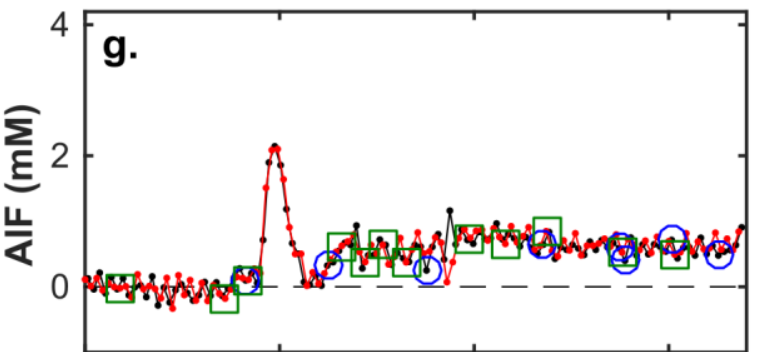

10

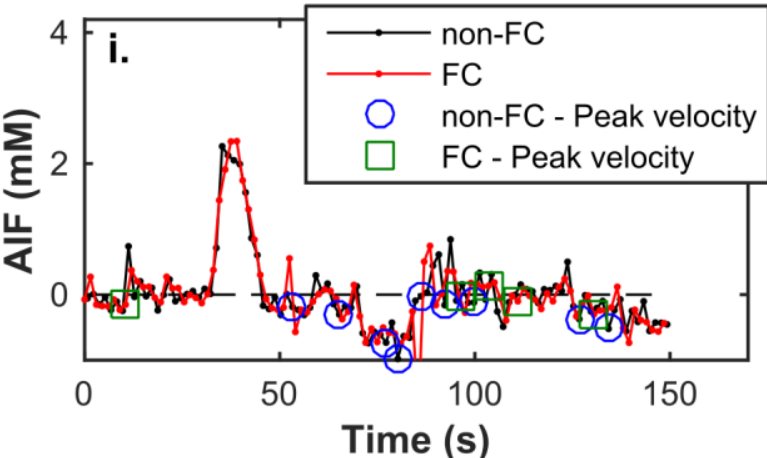

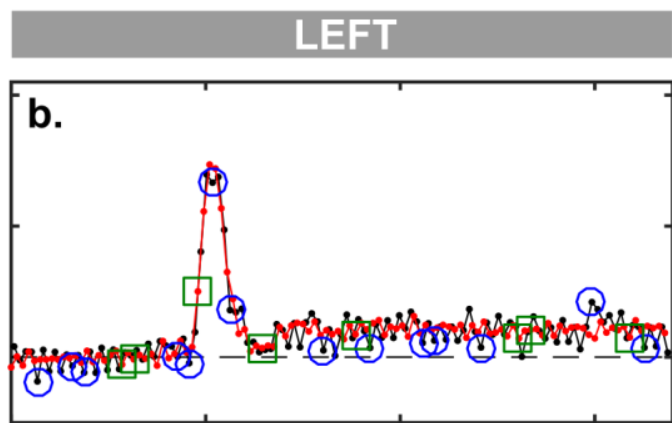
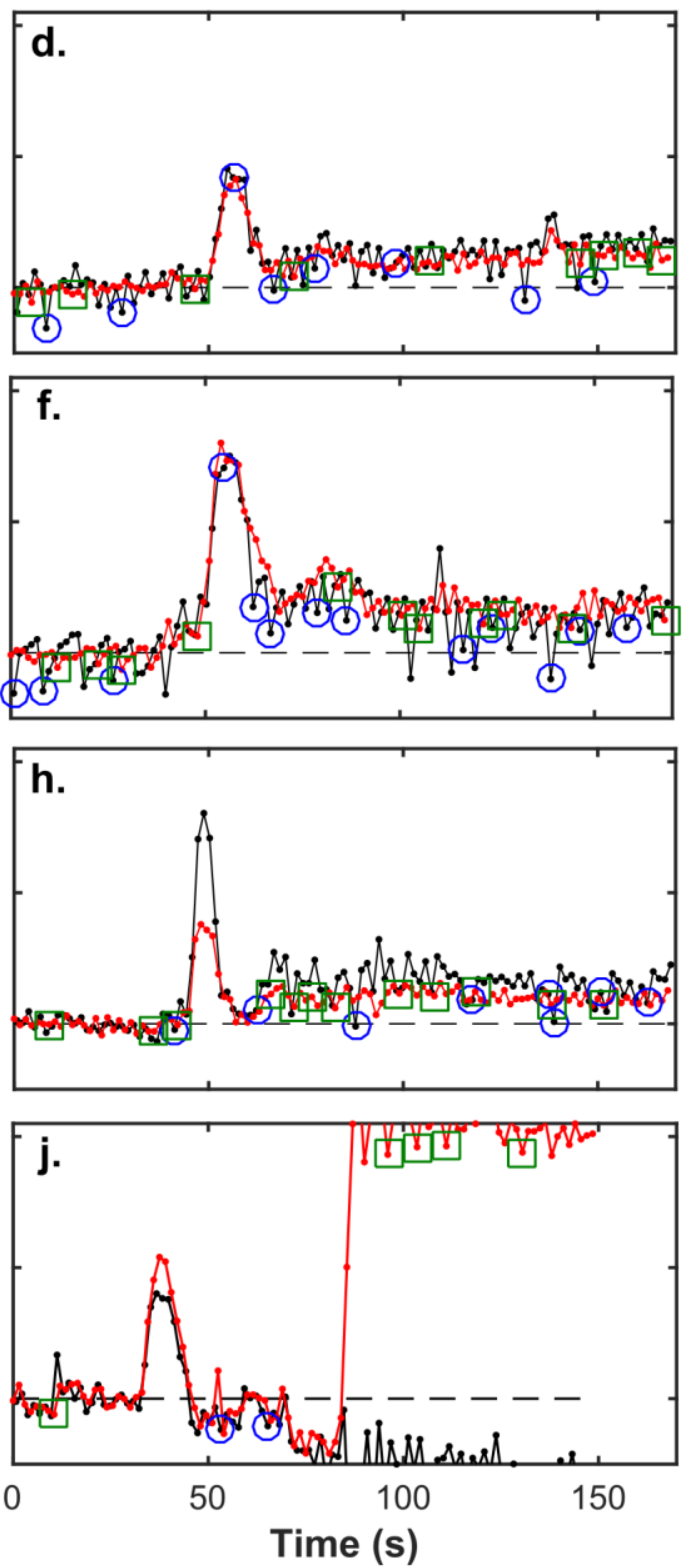

FIG. 6. Absolute quantification of the right and left ICA AIFs for all subjects. Right (a,c,e,g,i) and left $(b, d, f, h, j)$ partial volume corrected AIFs are displayed for non-FC and FC frames. Subject motion is responsible for the concentration shifts observed at $85 \mathrm{~s}$ for subject 5 AlFs (i,j). PVCM 
1 differences caused an increased shift for the left FC AIF (j). Peak velocity AIF points (open circles 2 and squares) are data points acquired within a trigger delay of 100-200 ms.

3 


\section{Subject 3 - LEFT}

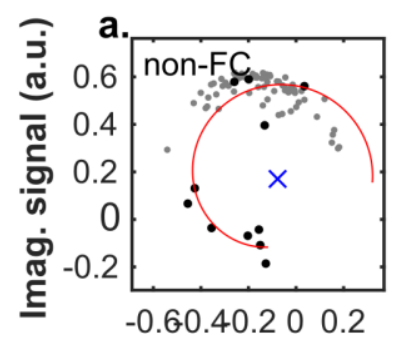

Real signal (a.u.)

$$
\text { e. }
$$

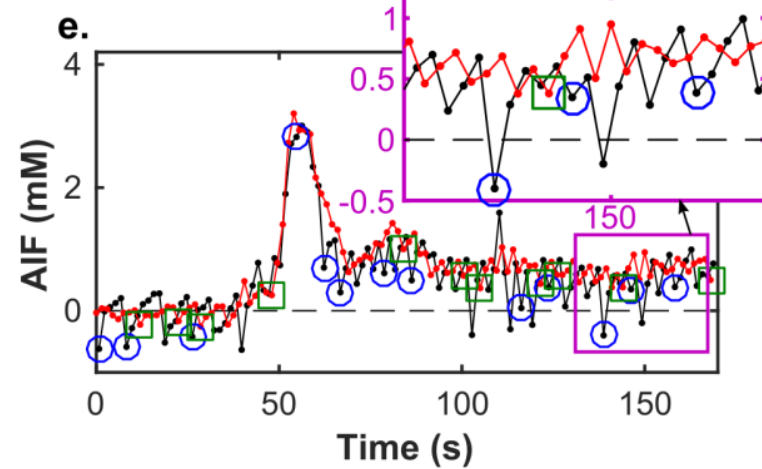

b.

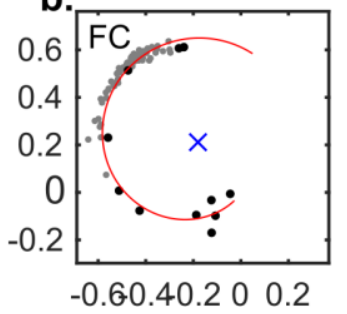

Real signal (a.u.)
Subject 5 - LEFT

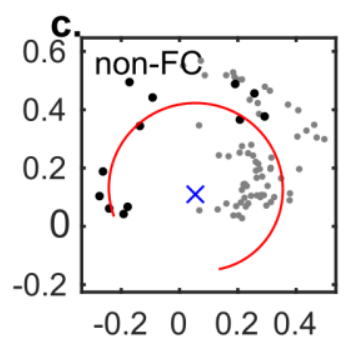

Real signal (a.u.)

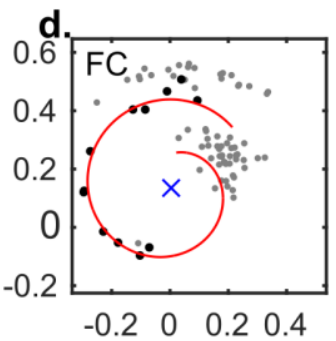

f.

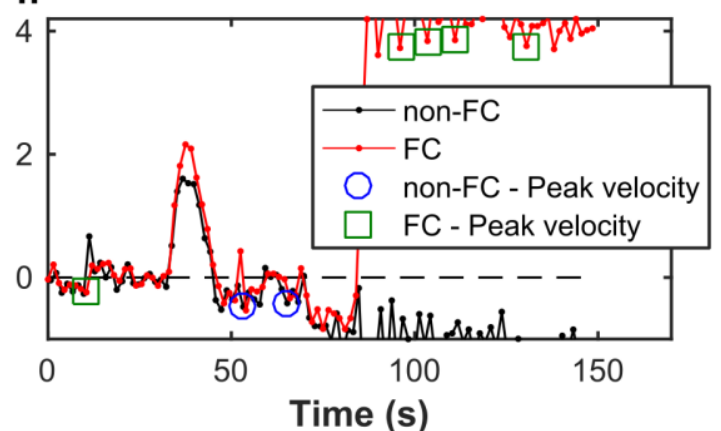

FIG. 7. Detailed comparison of non-FC and FC partial volume correction for cases with $(a, b, e)$ similar or (c,d,f) different fitted calibration curves. (a-d) Detailed PVCM for non-FC and FC average signal over the ICA. The dots represent the MR signal in the complex plane for all time frames. CA calibration curve spiral (red line), first pass time frames (black dots), and constant tissue signal (blue cross) are shown in the figure. $(e, f)$ partial volume corrected AIF are displayed for non-FC and FC frames. Subject motion is responsible for the concentration shifts observed at

$8 \quad 85 \mathrm{~s}$ for subject 5 AIFs (f). PVCM differences cause an increased shift for the FC AIF (red). Peak 9 velocity AIF points (open circles and squares) are data points acquired within a trigger delay of $100-200 \mathrm{~ms}$. 


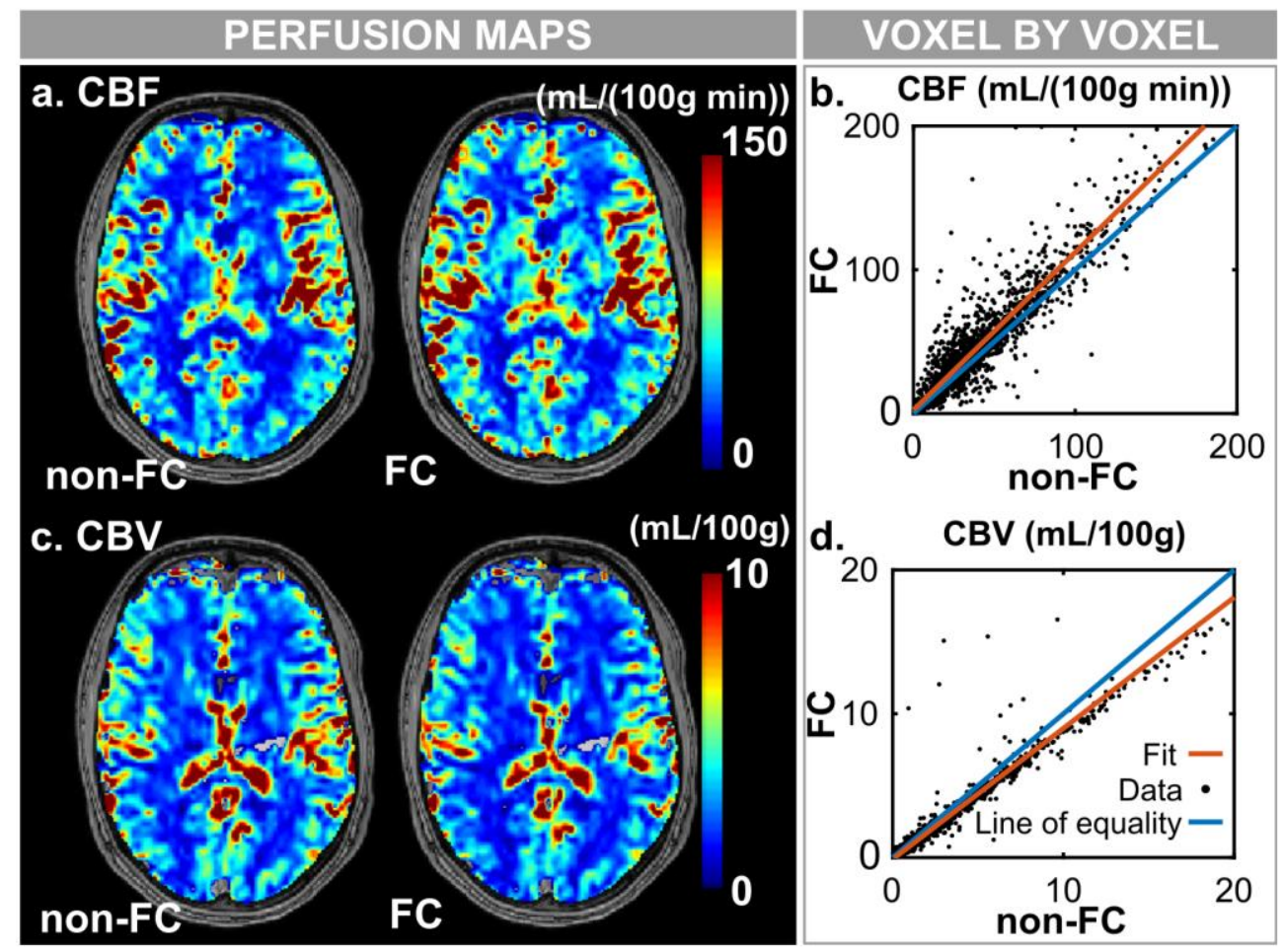

2 FIG. 8. (a) CBF and (c) CBV perfusion maps for non-FC and FC data with the AIFs from the left 3 ICA in Fig. 6f. Voxel by voxel comparison of (b) CBF and (d) CBV highlights the effect of FC on 4 perfusion metrics. The fitted slopes over (b) and under (d) the line of equality indicate that FC $5 \quad$ increases CBF and decreases CBV for this subject. 
Table 1. Mean CBF and CBV over gray matter (GM) and white matter (WM) ROls and percent differences of the voxel by voxel comparison $\left(D_{F C}\right)$ for the non-FC and FC perfusion experiments measured for the right and left AIF of all subjects. AIFs with major PVCM changes are underlined.

4 Subjects with significant increased CBF and decreased CBV in the voxel by voxel comparison 5 are highlighted in bold. $D_{F C}$ are considered significant when their $95 \%$ confidence interval (mean $6 \pm \mathrm{SD}$ ) excludes $0 \%$. Nonsignificant $D_{F C}$ are italicized.

\begin{tabular}{|c|c|c|c|c|c|c|c|c|c|c|c|}
\hline & & \multicolumn{4}{|c|}{ GM } & \multicolumn{4}{|c|}{ WM } & \multirow{2}{*}{\multicolumn{2}{|c|}{$D_{F C}(\%)$}} \\
\hline & & \multicolumn{2}{|c|}{ non-FC } & \multicolumn{2}{|r|}{$\mathrm{FC}$} & \multicolumn{2}{|c|}{ non-FC } & \multicolumn{2}{|c|}{ FC } & & \\
\hline \multicolumn{12}{|c|}{ CBF (mL/100g min), mean (SD) } \\
\hline \multirow[t]{2}{*}{ Subject 1 , } & Right AIF & 59 & $(47)$ & 65 & (53) & 39 & $(56)$ & 40 & (55) & 1.2 & (1.9) \\
\hline & Left AIF & 66 & (53) & 68 & $(57)$ & 43 & $(65)$ & 42 & (59) & -8.1 & $(2.0)$ \\
\hline \multirow[t]{2}{*}{ Subject 2, } & Right AIF & 66 & $(57)$ & 76 & $(82)$ & 24 & (33) & 26 & $(48)$ & 24.6 & (2.3) \\
\hline & Left AIF & 77 & $(67)$ & 103 & $(105)$ & 28 & (39) & 35 & (55) & 36.4 & (2.4) \\
\hline \multirow[t]{2}{*}{ Subject 3, } & Right AIF & 59 & $(48)$ & 71 & (61) & 24 & $(20)$ & 29 & $(26)$ & 17.6 & (2.3) \\
\hline & Left AIF & 64 & $(55)$ & 74 & $(62)$ & 26 & $(22)$ & 31 & $(27)$ & 9.7 & (2.0) \\
\hline \multirow[t]{2}{*}{ Subject 4, } & Right AIF & 106 & $(84)$ & 102 & (83) & 37 & $(32)$ & 35 & (29) & -19.3 & $(1.8)$ \\
\hline & $\underline{\text { Left AIF }}$ & $\underline{69}$ & $\underline{(52)}$ & $\underline{132}$ & $(106)$ & $\underline{24}$ & $\underline{(19)}$ & $\underline{46}$ & $\underline{(37)}$ & $\underline{91.6}$ & (3.1) \\
\hline \multicolumn{12}{|c|}{ CBV (mL/100g), mean (SD) } \\
\hline \multirow[t]{2}{*}{ Subject 1, } & Right AIF & 3.2 & $(2.7)$ & 3.1 & $(2.6)$ & 2.1 & (2.0) & 2.1 & $(2.1)$ & -3.1 & $(1.0)$ \\
\hline & Left AIF & 3.4 & (2.9) & 3.3 & $(2.8)$ & 2.3 & $(2.2)$ & 2.2 & $(2.2)$ & -4.3 & (1.0) \\
\hline \multirow[t]{2}{*}{ Subject 2, } & Right AIF & 3.7 & $(4.1)$ & 3.3 & $(3.7)$ & 1.8 & $(2.0)$ & 1.6 & $(1.8)$ & -13.7 & (1.2) \\
\hline & Left AIF & 3.6 & $(4.0)$ & 3.8 & $(4.2)$ & 1.7 & (1.9) & 1.9 & (2.1) & 1.3 & (1.5) \\
\hline \multirow[t]{2}{*}{ Subject 3, } & Right AIF & 4.2 & (3.8) & 3.6 & (3.3) & 1.9 & $(1.7)$ & 1.7 & (1.5) & -8.5 & (2.4) \\
\hline & Left AIF & 3.9 & (3.6) & 3.4 & (3.1) & 1.8 & (1.6) & 1.6 & (1.4) & -8.9 & (2.3) \\
\hline \multirow[t]{2}{*}{ Subject 4, } & Right AIF & 4.5 & (3.4) & 4.4 & (3.5) & 2.4 & (1.8) & 2.3 & $(1.7)$ & -4.2 & $(1.0)$ \\
\hline & Left AIF & $\underline{3.6}$ & (2.7) & $\underline{6.0}$ & $(4.7)$ & 1.9 & (1.4) & 3.2 & (2.3) & $\underline{64.8}$ & (1.7) \\
\hline
\end{tabular}




\section{Supporting information}

\section{A. Flow effect simulations for the SS-EPI sequence}

Flow effects were simulated with an infinite tube model with constant-velocity parabolic flow based on the velocity k-space formalism. ${ }^{1}$ The effects of constant-velocity flow are the only source of signal variation considered in the simulation. $\mathrm{T}_{1}$ relaxation, $\mathrm{T}_{2}^{*}$ relaxation, higher order flow effects, partial volume effects and inflow effects were neglected. Our implementation of the velocity k-space formalism was validated by reproducing the results of Nishimura et al. ${ }^{8}$ The scanner SS-EPI sequence waveform was imported into the simulation framework. Default EPI ramp sampling was removed from the sequence to allow Cartesian sampling of k-space. The tube simulation parameters were chosen to match the geometry and flow velocity of the MCA (diameter of $3 \mathrm{~mm}$, pulsatile flow velocity data from one subject, arterial angulation at $45^{\circ}$ with the phase encoding gradient $\left(G_{p}\right)$ ).

Fig. S1a shows the simulated image of an infinite tube with constant velocity parabolic flow for the SS-EPI sequence. Simulations were repeated for representative input velocities for 15 phases of the cardiac cycle. The resulting signal magnitude and phase are plotted against trigger delay for the non-FC and FC sequences (Fig. S1bc). For the standard non-FC sequence, the signal magnitude is decreased by as much as $20 \%$ and the signal phase is increased by $30^{\circ}$ when the blood velocity is maximum. These variations result from the changing constant-velocity flow SS-EPI echo train partially recovers these variations. 
a.
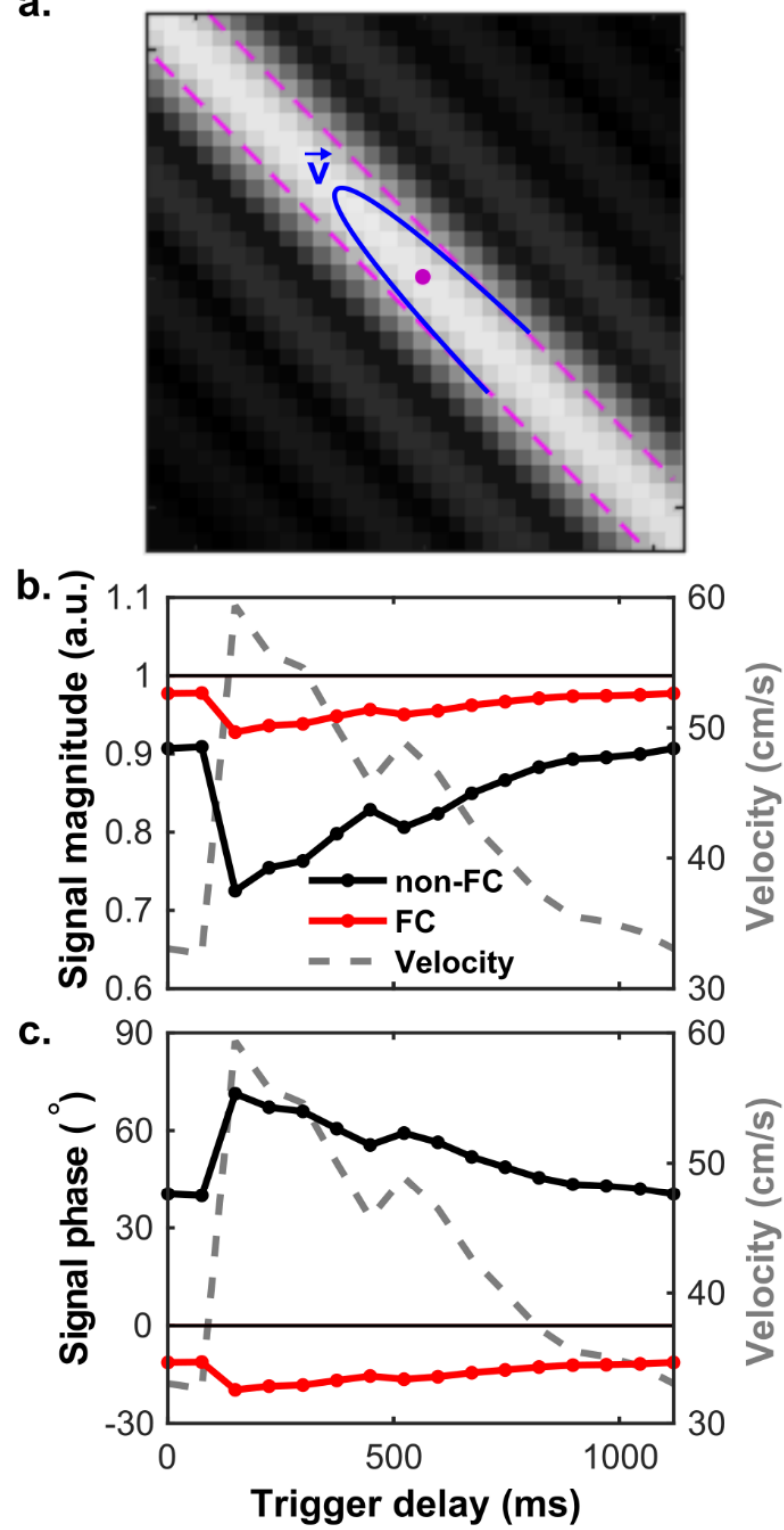

2 Supporting Information FIG. S1. Simulations of constant-velocity flow effects for the MCA show 3 signal variations caused by the pulsatile flow velocity. (a) Image of the modeled tube with the tube 4 wall location (dashed lines), the pixel chosen for analysis (dot), and the parabolic velocity profile 5 (line) for the non-FC SS-EPI sequence and a diastolic velocity of $33 \mathrm{~cm} / \mathrm{s}$. (b) Normalized signal 6 magnitude and (c) phase curves simulated for different flow velocities (dashed curve) associated 7 with their trigger delay. The FC SS-EPI sequence partially recovers the signal magnitude and $8 \quad$ phase variations caused by pulsatile velocity. 


\section{B. SS-EPI with a $30^{\circ}$ flip angle}

The $R_{\mathrm{SNR}}, R_{\mathrm{SD}}$, and correlation metrics for MCA ROI 3 and ICA were calculated for every subject that was scanned with the $30^{\circ}$ flip angle sequence (Fig. S2). With limited tissue saturation for this reduced flip angle, the blood signal was not hyperintense on these datasets. Thus the ROI definition could not rely on voxels with high signal values. We selected voxel following the vessel anatomy and showing maximal velocity correlated signal variations for the non-FC dataset. This is expected to select the voxels with maximal blood signal content because these velocity correlated signal variations matched the high blood signal intensity on our previous experiment with the $90^{\circ}$ flip angle. However, the flow effects are maximized by this voxel selection scheme and could lead to an overestimation of the effect of FC shown in Fig. S2.

The data of Fig. 5 used ROI defined from the hyperintense blood signal at $90^{\circ}$ flip angle and should be a more objective representation of FC effects. Both figures show similar FC effects, with reduced sample size and $p$-values for Fig. S2.
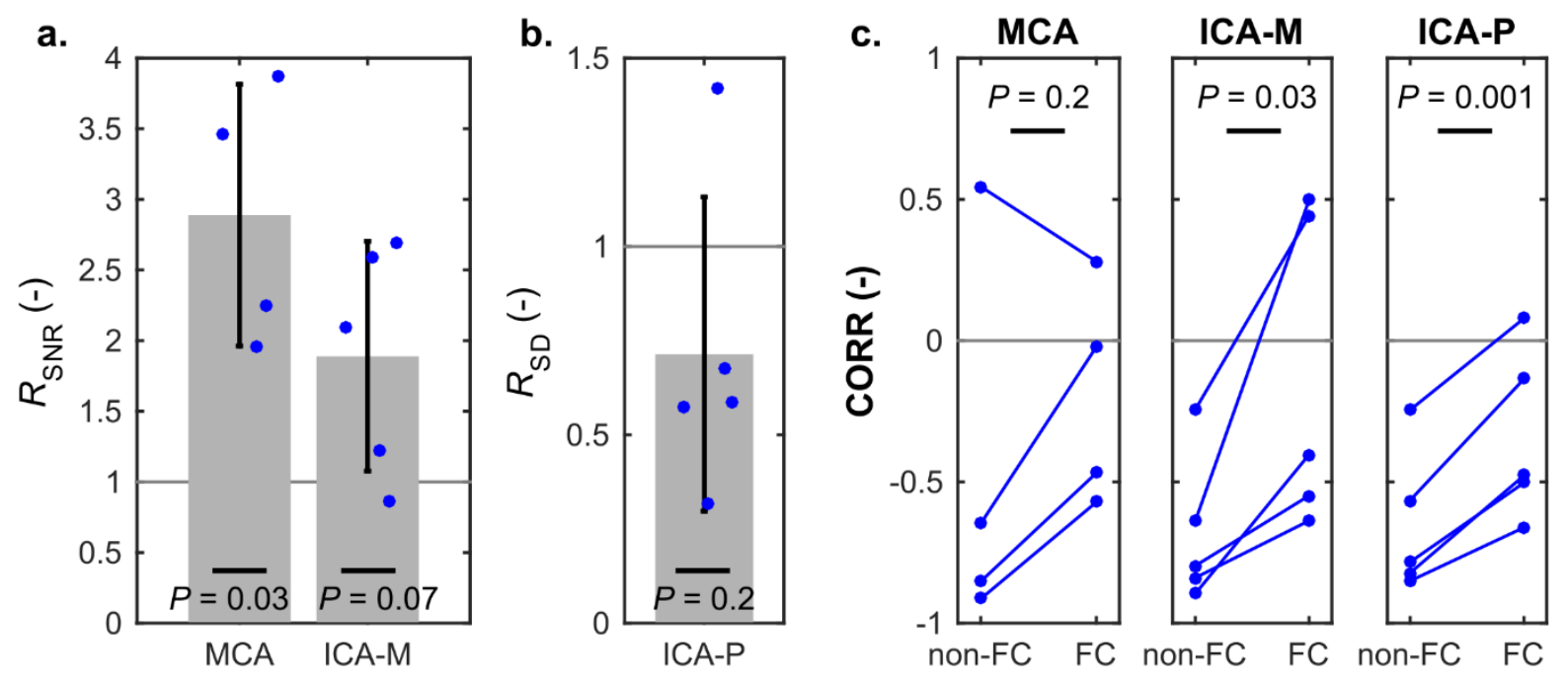

Supporting Information FIG. S2. Effect of FC on SS-EPI with a $30^{\circ}$ flip angle (a) SNR ratio $\left(R_{\mathrm{SNR}}\right),(\mathrm{b})$ (b) phase $\mathrm{SD}$ ratio $\left(R_{\mathrm{SD}}\right)$, and (c) correlation between signal and flow velocity (CORR). These metrics are compiled for all subjects MCA $(\mathrm{N}=4)$ and ICA $(\mathrm{N}=5)$. ICA-M and ICA-P indicate the metrics calculated for ICA signal magnitude and phase, respectively. $p$-values are shown for all datasets. 
In Fig. S3 we report the results of the subject with the lowest SNR measured in the MCA. We note a pronounced vessel curvature all along the MCA (Fig. S3a) associated with important signal variations inversely correlated with blood velocity (Fig. S3b); those variations are virtually identical for the FC acquisition.

a.

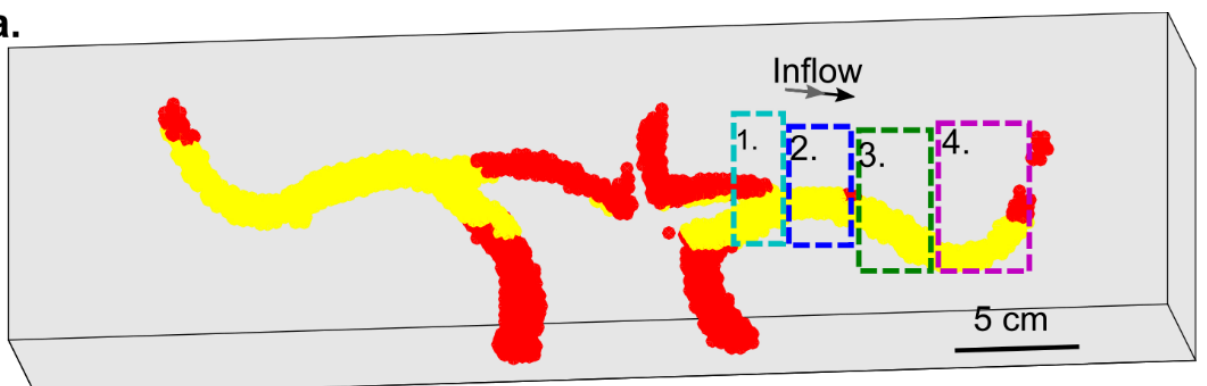

b.
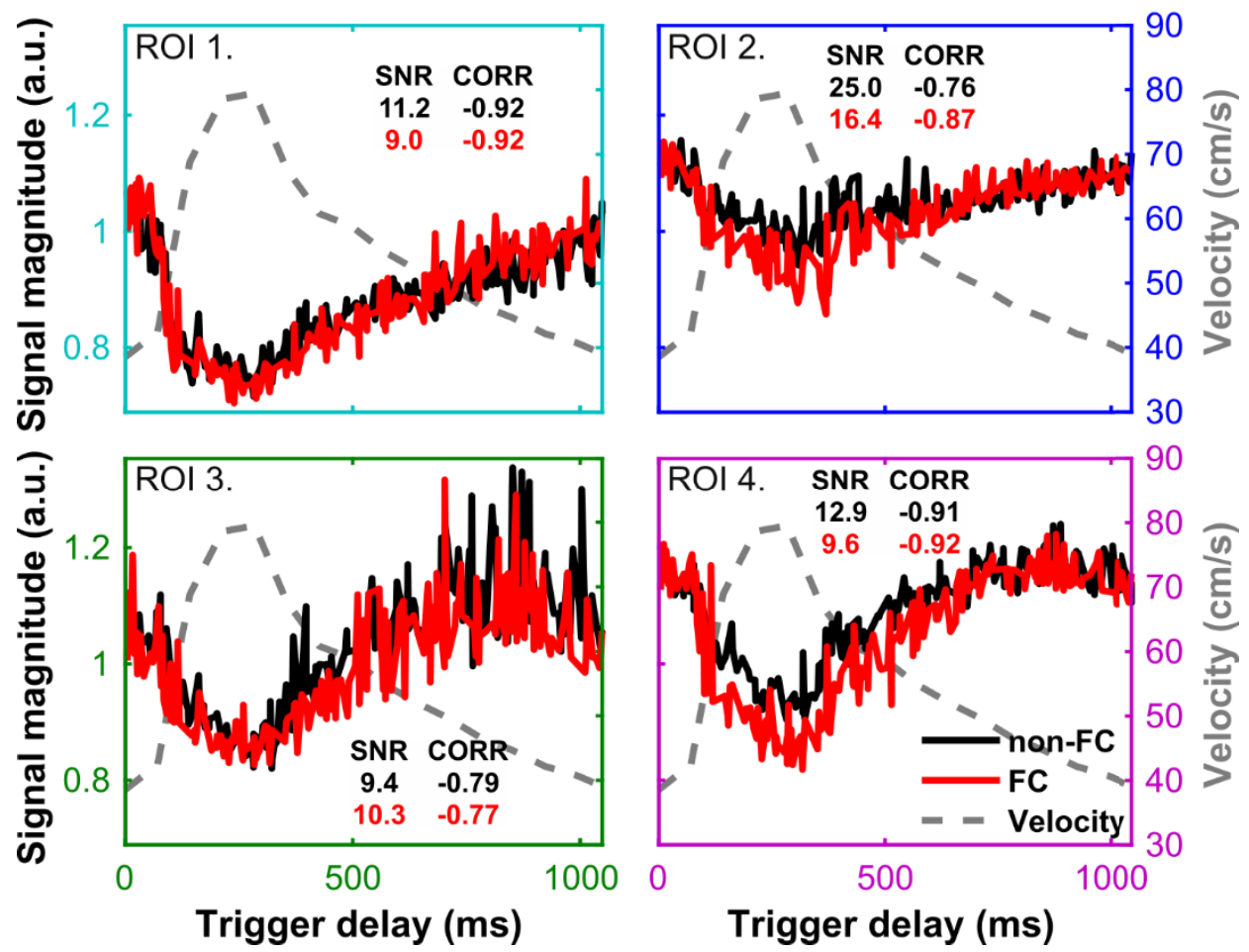

Supporting Information FIG. S3. An example of poor FC performance for a subject with curved MCA. (a) An enlarged section of the MCA blood mask (as in Fig. 1b) shows high vessel curvature and unexcited blood (red points) between ROI 2 and 3. (b) Dynamic first echo signal sorted by trigger delay for each ROI of this MCA for FC and non-FC SS-EPI sequences with measured MCA blood flow velocity (dashed curve). SNR and correlation between signal and velocity (CORR) are indicated for each ROI. 


\section{Flow effect simulations for the SS-EPI sequence for poorly aligned ICA}

Realistic flow effect simulations for the ICA require a complex model with finite excitation length limited by the slice profile. The flow effect simulations of the infinite tube shown in 4 Supporting Information S1 are not a realistic representation of an artery that crosses the slice 5 plane. Nevertheless, we used this model as a first level of approximation to investigate the 6 magnitude of transverse flow effect for a misaligned ICA.

To do so, the projection of the ICA in the transverse plane is approximated as an infinite tube with parabolic flow. For an ICA aligned with $G_{s}$ at $16^{\circ}$ (the top $20 \%$ misaligned ICA in our study are over $16^{\circ}$ ), the magnitude of the transverse velocity reaches over $30 \%$ of the total velocity. With ICA pulsatile velocities ranging from 30 to $80 \mathrm{~cm} / \mathrm{s}$, this corresponds to an in-plane velocity variation of 9 to $24 \mathrm{~cm} / \mathrm{s}$.

The simulated non-FC inplane flow effects were small for signal intensity variations (0.95$0.99)$ and for important phase variations $\left(11-28^{\circ}\right)$. The phase variations represent an error of 0.25 $\mathrm{mM}$ for phase based AIF measurements, wich is about $10 \%$ of the reported AIF peaks. FC could recover most of this variation. This simulation suggests that $G_{f}$ and $G_{p} F C$ provides an important noise reduction for the poorly misaligned ICA.

The slice dephasing was not included in this simulation, thus both FC and non-FC curves

would be perfectly flow-compensated for $\mathrm{G}_{\mathrm{s}}$. Our simulation framework cannot estimate the corresponding noise component to the $F C$ in the $G_{s}$ direction and would require a more complex model of a finite excited tube limited by the slice profile. Thus we cannot comment on the relative improvement of $F C$ in all directions compared to a $G_{s}$-only $F C$, this simulation provides a first level of approximation limited to the absolute improvement of inplane flow effects by FC in all directions. 


\section{E. Flow effect simulations for the 3D SPGR sequence}

A dual echo 3D SPGR sequence waveform was imported from the scanner into the simulation framework with and without flow compensation. The sequence diagram is shown in Fig. S4a for the FC gradients waveforms. The sequence had the additional following parameters: FOV $=220$ x $158 \times 90 \mathrm{~mm}^{3}$; spatial resolution $=1.7 \times 1.7 \times 3 \mathrm{~mm}^{3}$; time resolution $=33 \mathrm{~s}$; Profile order: CENTRA. Parallel imaging acceleration was removed from the sequence to allow Cartesian sampling of k-space.

The simulation framework described in Supporting information S1 was modified for 3D acquisition. In this case, the dynamic frame k-space is sampled over several cardiac cycle. The specific velocity of each line of k-space was found with sequence timings. To do so, the subject velocity profile was first sub-sampled with 16 specific velocities (Fig. S4b). Then, the constantvelocity 3D k-space was simulated for each of these velocities with the parabolic flow infinite tube model. Next, the frame k-space with pulsatile velocity was assembled using data from these constant-velocity k-spaces, where sequence timings and profile ordering were compared to a constant cardiac cycle to attribute the velocities in the frame phase-encoding plane (Fig. S4c). This was repeated for 16 frames with different starting trigger delays in the cardiac cycle. The signal magnitude and phase from the voxel with the highest magnitude in the centered slice (Fig. S4d - voxel identified with the dot) is reported for the 16 frames (Fig. S4ef).

The signal loss from flow effects is constant and induced by the displacement in the $k_{z}$ phase encoding direction that was not accounted for by voxel selection. This effect would result in a constant SNR loss and increased PVE. 

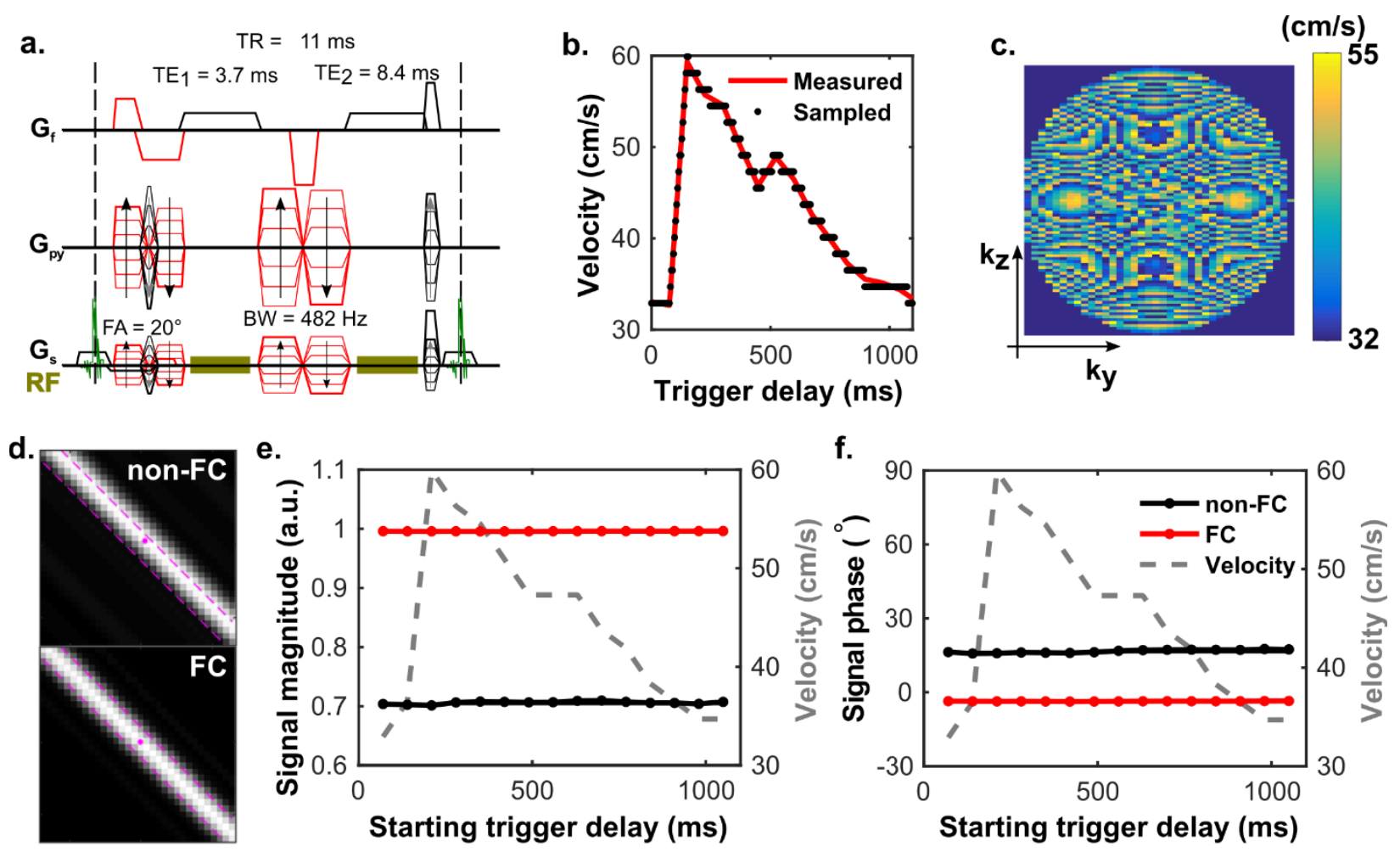

Supporting Information FIG. S4. Simulations of flow effects for the MCA show a constant flow effect caused by the pulsatile flow velocity. (a) FC dual-echo 3D SPGR sequence diagram with the FC specific gradients identified in red. (b) Sub-sampling of the MCA velocity profile. (c) Velocities in the $k_{y}-k_{z}$ plane for the time frame with a starting trigger delay of $70 \mathrm{~ms}$. (d) Image of the modeled tube with the tube wall location (dashed lines) and the pixel chosen for analysis (dot) for the first frame of the non-FC and FC sequence. (e) Normalized signal magnitude and (f) phase curves simulated for different flow velocities (dashed curve) associated with their starting trigger delay.

\section{References}

1. Nishimura DG, Irarrazabal P, Meyer CH. A Velocity k-Space Analysis of Flow Effects in Echo- Planar and Spiral Imaging. Magn Reson Med. 1995;33:549-556. 